Original Article

\title{
A method of seeing in homeopathy: methodological foundations of project "Understanding Homeopathy by Images"
}

\author{
Gheorghe Jurj \\ West University, Timisoara, Romania
}

\begin{abstract}
This paper presents the results of conceptual research on the nature and operation of visual signs in homeopathic clinical practice. Aims: to analyze the role of visual signs in homeopathy together with their specific semiotic structure in correlation with the codes leading to possible significations and then to assess the possibility of establishing coherence between the symptoms and signs of patients. Methodology: Sign-complexes are approached through a four-stage model including: 1) delimitation; 2) decomposition; 3) correlation and; 4) reintegration of signs. Data for analysis were collected along 15 years of homeopathic clinical practice and recorded by visual means; theoretical foundations derive from Jakobson's theory of communication and Peirce's semiotics. Results: stages of delimitation and decomposition are illustrated by a homeopathic semiology of the tongue; analysis of correlation allowed to identify 9 different possible patterns; reintegration requires a first phase of extension of the connotation and denotation of signs followed by a phase of contraction of meaning leading to decision-making. Conclusion: application of the model proposed results in highly qualified and individual visual signs that bring new elements to reconsider the role of objective signs in homeopathic practice.
\end{abstract}

Keywords: Homeopathy; Semiotics; Semiology; Visual signs

\section{Introduction}

In medicine, knowledge of the state of patients is possible through a cognitive process mediated by signs. In fact, signs are the elementary units to approach any aspect of reality involving a sending and receiving of information [1]. Whereas from the cognitive viewpoint, medicine as a whole is a semiotic (i.e. sign-mediated) process, homeopathy is much more so as it explicitly operates at the level of perceptible phenomena.

Signs have been classified as verbal and non verbal, in medicine this corresponds to "symptoms" and "signs", respectively. Homeopathy has historically prioritized verbal communication, as it deals with subjective data, held as foremost for the individualization of each particular patient for prescribing purposes. However, a deeper analysis as recently undertaken by semioticians shows that verbal communication is a highly complex mode of communication involving many factors that can distort the information conveyed by patient [2]. On the other hand, vision is a primary and spontaneous, unmediated channel of communication, supplying direct evidence (from the Latin, e-videre: "to see something as it really is") on the state of patients. For this reason, visual signs must be an integral part of the "totality of symptoms" on which homeopaths ground the choice of remedies.

The aims of this paper are first to analyze the role of visual signs in homeopathy together with their specific semiotic structure in correlation with the codes leading to possible significations and then to assess the possibility of establishing coherence between the symptoms and signs of patients.

\section{Methodology}

The semiotic model proposed includes four general steps: 1) Delimitation, or identification of breaks in the continuity of reality; 2) Decomposition, or analysis of signs as actually perceived into semiotic units as a function of predetermined axes allowing to qualify signs through their components and relationships; 3) Correlation between a) visual and verbal signs, b) homeopathic clinical signs and homeopathic codes of interpretation (repertories, materia medica, etc.), c) between clinical signs and homeopathic remedies; 4) Reintegration, involving the synthesis of the earlier analyzed signs within 
the context of the totality of signs exhibited by the patient according to the principle of coherence.

Sign-complexes, which I call "configurations", are approached according to the four-stage model described above. Data were collected along 15 years of clinical homeopathic practice in thousands of patients and recorded by visual means (photography and video) within a larger project entitled "Understanding Homeopathy by Images" of which this paper is a methodological introduction [3] Analysis is grounded on Roman Jakobson's theory of communication and Charles S. Peirce's semiotics [57].

\section{Results}

An element in reality is perceived as a sign if: 1) it is delimitated from the continuum of reality; 2) it is located within a given context; 3) then it is demarcated from its context through an operation of differentiation; and 4) it is linked to a definite (actual or potential) meaning

Semiology of the tongue can be used as a case-study to illustrate this process. In this regard, it must be reminded there is a difference between the pathology of the tongue, with its corresponding pathological signs leading to diagnosis of diseases, and the signs of the tongue as indicators of remedies, that is to say, the tongue as a possible field of expression of homeopathic remedies and individual patterns of sensitiveness and reactivity. Hence, regarding pathology, corresponding rubrics in the homeopathic repertories include: complaints of the tongue; pain in the tongue; inflammation; ulcers; aphthae; cancer, etc. On the other hand, non pathological rubrics, indicating modes and states of being are related to features such as the general appearance of the tongue, its coloration, presence of cracks, so-called mapped tongue, etc.

There are several levels in approaching the tongue as a carrier of meaning in homeopathy, i.e. as indicating individuality. As in the general model described in this paper, the tongue may be approached: 1) as a whole; 2) in its elements; 3) in the correlation of its elements; 4) in the correlation of its elements with concomitant signs and symptoms in other areas.

As a whole, the first item to be approached is the general appearance of the tongue in both its static and dynamic dimensions. Static features include: thickness; size; width; degree of trophism; cleanness; covering; cracks; surface, etc. Dynamic features include trembling, motions, retractions, deviations, paresis, paralysis, etc. This stage can supply many useful data, a part of which is already codified in the homeopathic repertory and materia medica, however the lion's share still awaits to be codified.

Regarding the elements of the tongue, once again there are two levels: what is seen and where, i.e. what kinds of modifications can be perceived in relation to particular structures or areas. The question on space alludes to: 1) anatomical level: flesh, mucosa, coating; 2) topography: centre, margin, tip, root; axes of analysis are illustrated in Figure 1.

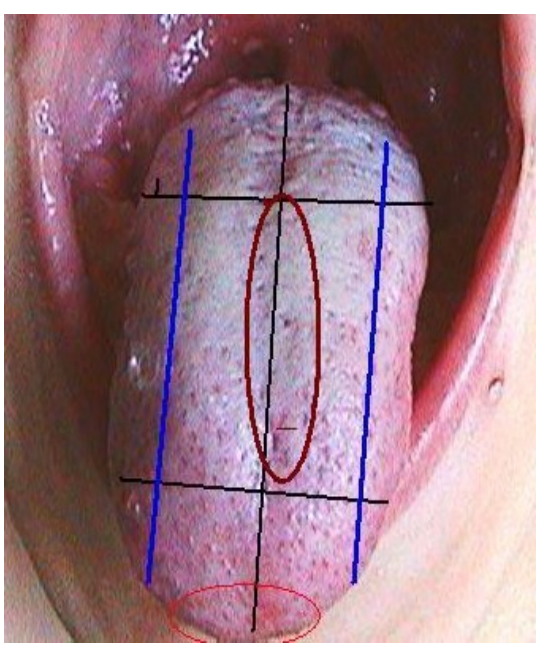

Figure 1. Regarding now what can be seen, it is useful to keep in mind that initial inspection requires a non interpretative stare: this is a moment allowing for the detection of potential signs, i.e. anything existing that might be homeopathically meaningful.

Initial inspection in Figure 2, e.g. reveals the tongue cracked in all directions with transversal deep fissure ; on the tip of tongue a depapillated area can be seen as well as the imprints of the teeth (this configuration can be suggestive of Calc-f, Fl-ac, Nit$a c$, Sil, remedies derived from iodine).

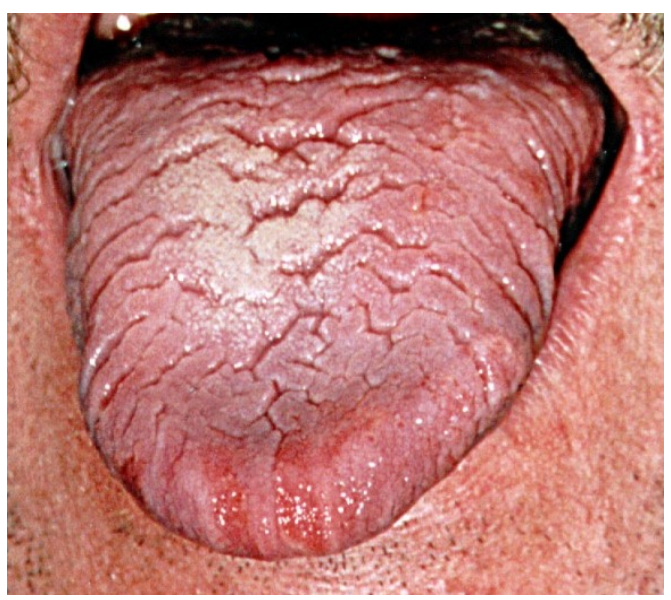

Figure 2 


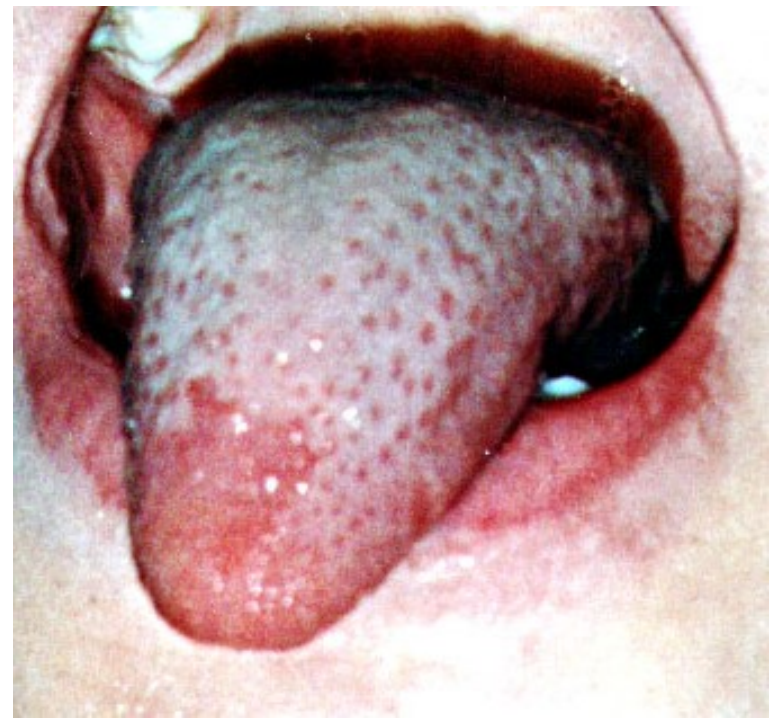

Figure 3

In Figure 3 it can be seen a "mapped" appearance: the right side of the tip is depapillated, whereas the rest of the tongue is covered by a white coating and the papillae are erect ( this image is frequently associated with Rhus-t and muriatic remedies, such as this case of Nat-m).

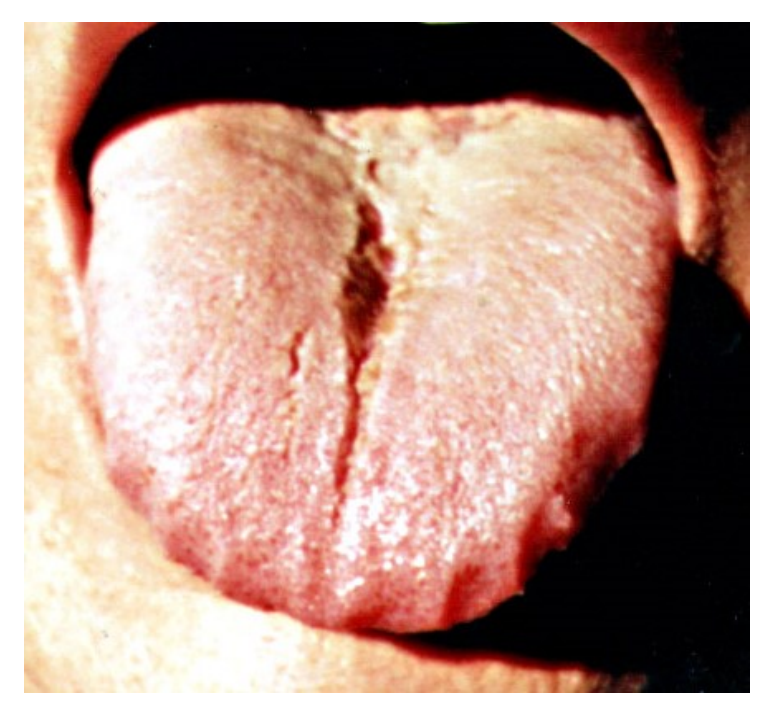

Figure 4

In Figure 4, the tongue has a yellow-brownish coating, more on the middle and posterior parts; there is a large and deep longitudinal fissure on the middle line and some small paramedian cracks; to notice also the deep imprint of the teeth all around the lateral and anterior part of tongue (this configuration is highly indicative of the mercury class of remedies, however it can also be found in Rhus-t, Ars, Chel, Sep, Iod, Baryta remedies, etc.)

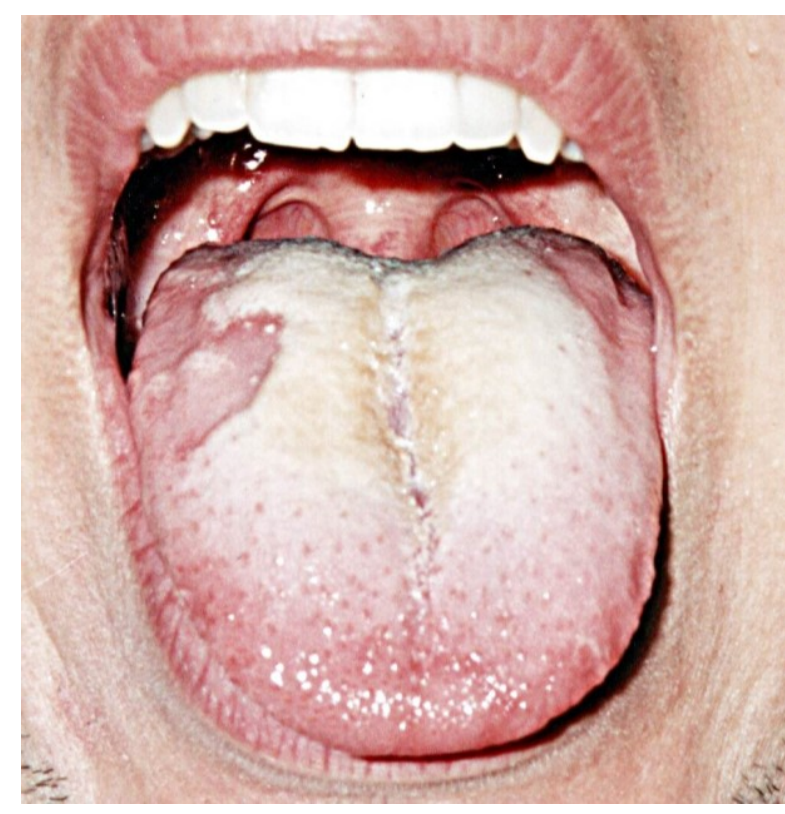

Figure 5

Figure 5 also shows a "mapped tongue", but here depapillation affects the margins of the tongue, which is also flabby and laterally indented; coating is white and furry thick and there is a sinuous crack in the middle line (this one was a case of Bar-m).

It must be emphasized that it is never a matter of one single and isolated sign the one that points to possible remedies. Figure 6 (a,b) shows two instances of "scrotal tongue", appearing respectively in patients sensitive to Natrum muriaticum and Silicea terra, respectively.

Particular configurations of local signs systematically involve several levels in correlation: coating, cracks, indentation, state of the papillae, etc. as well as their reciprocal relations. In all homeopathic configurations, the more accurately described are the features of structures and their correlation with the totality of signs and symptoms, the more narrows the scope of possible remedies and consequently, the more increases the reliability of a prescription.

For instance, in Figure 7, the semiotic value of the signs exhibited by the tongue does not come from any isolated trait, but from the relationship of opposition between the thick white (toxic) coating and the clean purple-red margin (Lac-c, Merc, Phyt). 

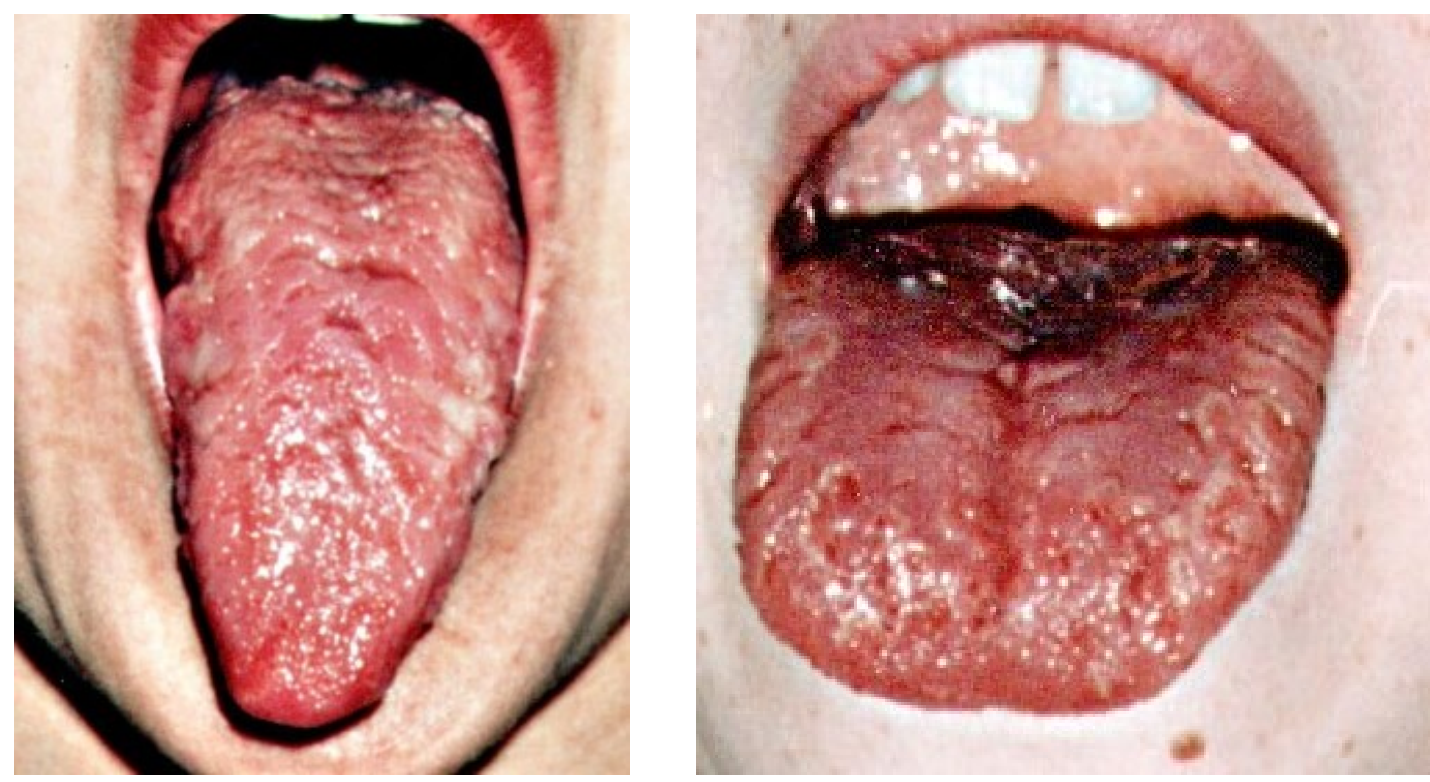

Figure $6(a, b)$

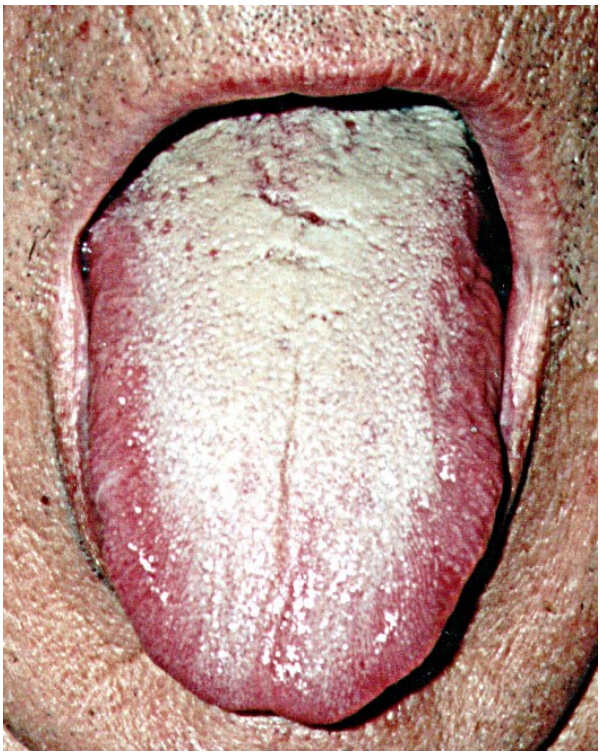

Figure 7

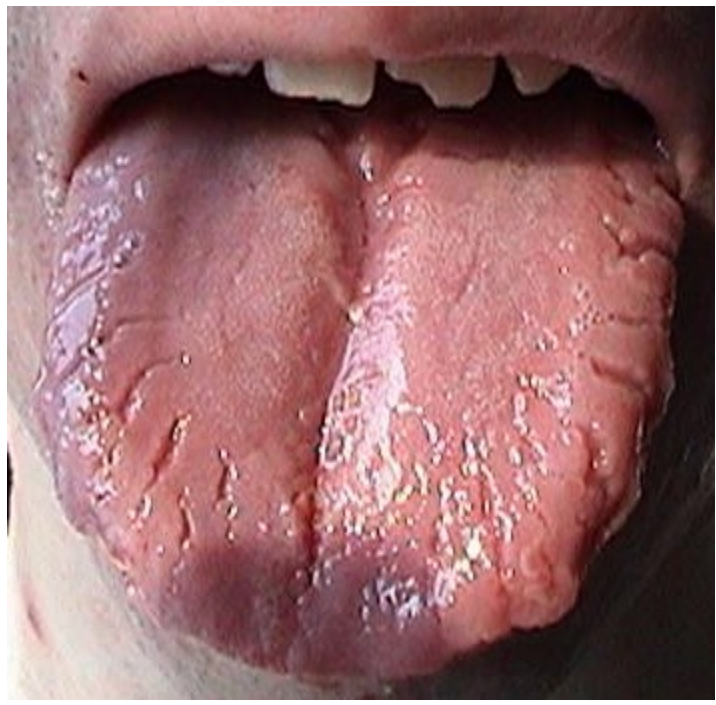

Figure 8

Correlation between the features of cracks and the appearance of the teeth is illustrated in Figure 8: radial cracks on the margins; deep middle line fissure which is sinuous and asymmetric; broken and malaligned teeth (fluoride remedies).

Figure 9 shows a fully depapillated tongue, with slight mostly longitudinal cracks on the surface of the tongue and a white area of leukoplasia on the right margin; the margins present deeper transversal cracks (here, a case of Alum, however fully depapillated tongue also appears in Ars and Ter). 


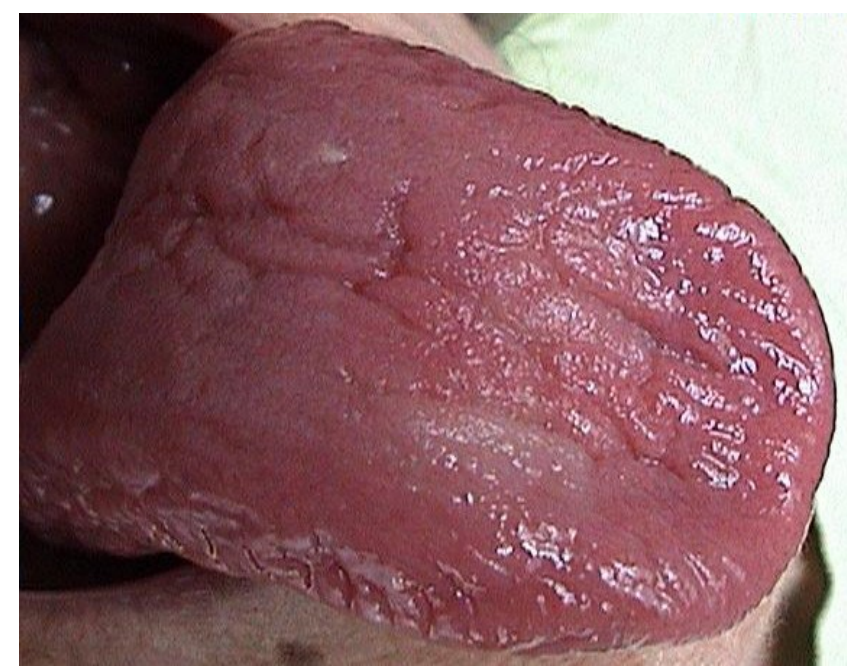

Figure 9

The tongue in Figure 10 is also depapillated, and there are white areas of dysplasia as well as superficial excoriations and wide ulcerations - large but not too deep - on a congestive base; the tongue as a whole is increased in sized and swollen (Caps).

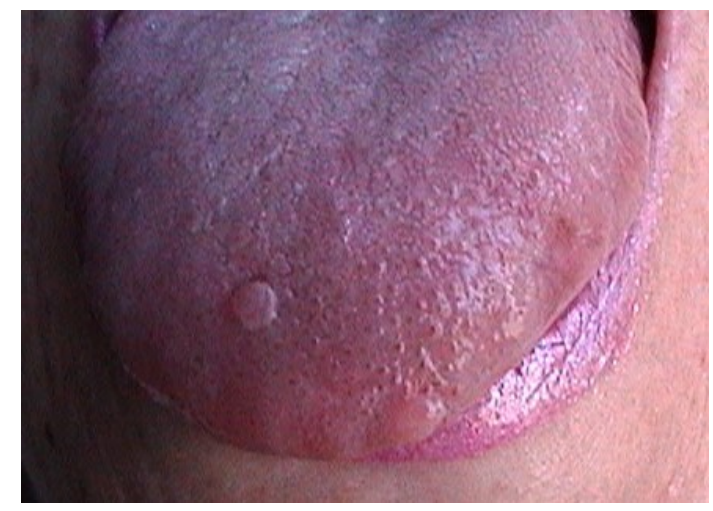

Figure 10

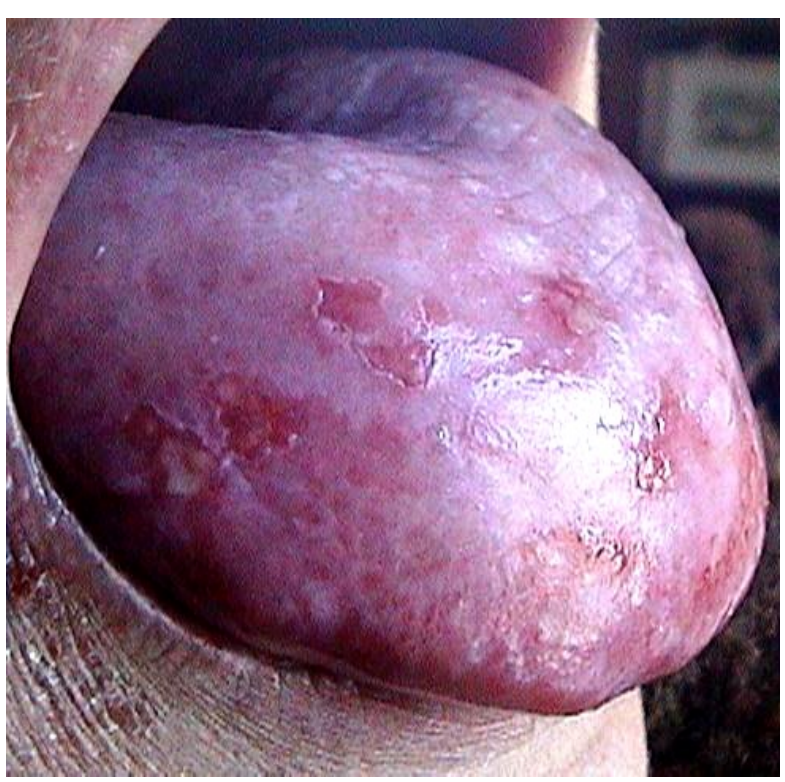

Figure 11
Sometimes, pathological growths can convey significant information. Figure 11 shows two warts on a flabby cyanotic tongue (narrowing the scope of remedies, as in this case, to Lach, where tongue warts are large and round, as shown in this image).

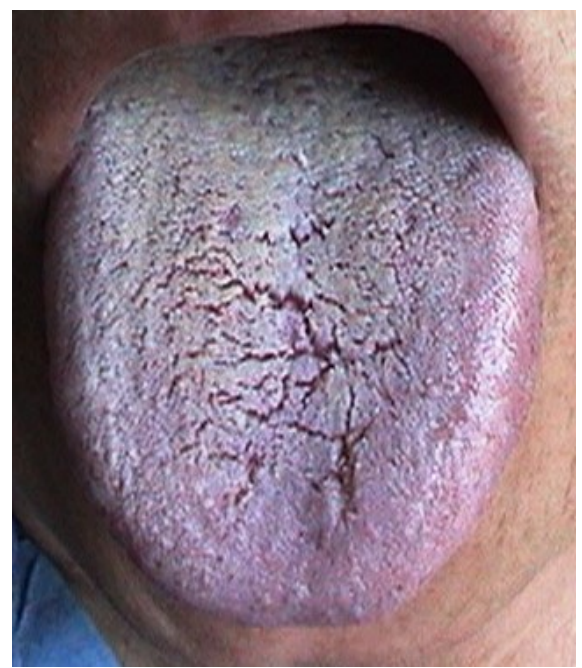

Figure 12

The basic configuration associated with Lachesis (broad, flabby and cyanotic tongue) reappears in this other patient (Figure 12), this time together with a bluish thick coating cracked in all directions.

The semiotic elements of the tongue can also correlate to structures outside the mouth. In Figure $13(\mathrm{a}, \mathrm{b})$, the tongue is deviated to the right and depapillated in correlation with the many warts on the eyelids and wrinkled face within the context of a Causticum configuration.
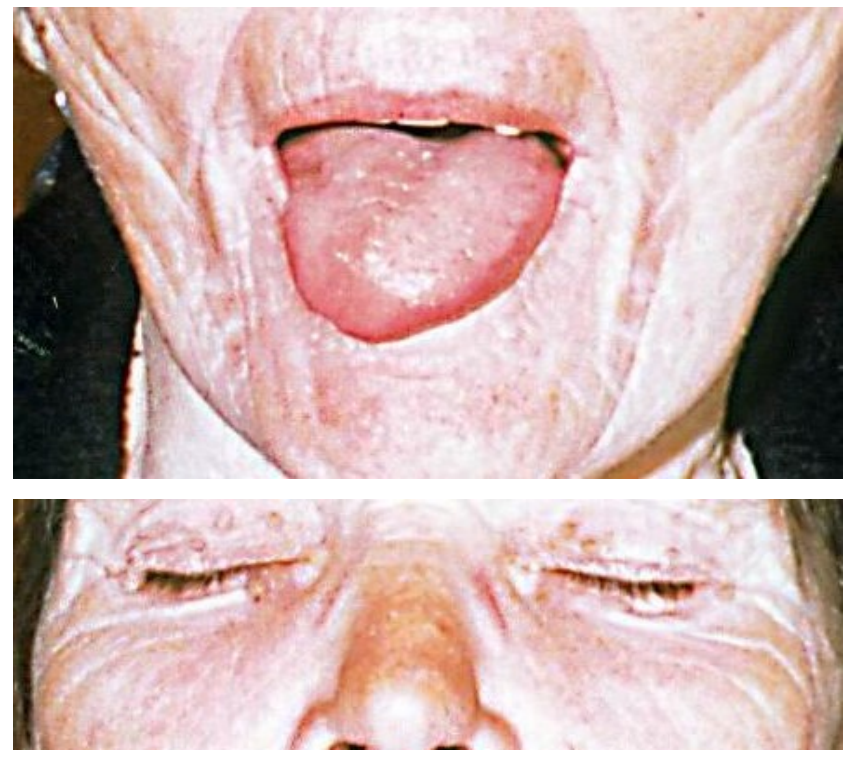

Figure 13 
The description of visual sign in homeopathic codes (viz. repertories and materia medica) sometimes requires multiple linguistic elements, as illustrated in Figure 14 (a case of Sul-ac). On the other hand, visual signs in one area correlate to symptoms corresponding to the same or other areas.

Visual signs have power of self-evidence, thus, they comply with the principle of consistence. Nevertheless, isolated signs never represent the totality of the individual, but they must be associated in configurations of signs and symptoms, from where follows the principle of coherence, which correlates various signs and symptoms in coherent wholes [1].

Figure $15 \quad(a, b)$ illustrates a configuration of Belladona: red tongue with erect papillae ("strawberry tongue" correlated with scarlatiniform rash over the body, high fever attended with torpor, dry skin and mydriasis.

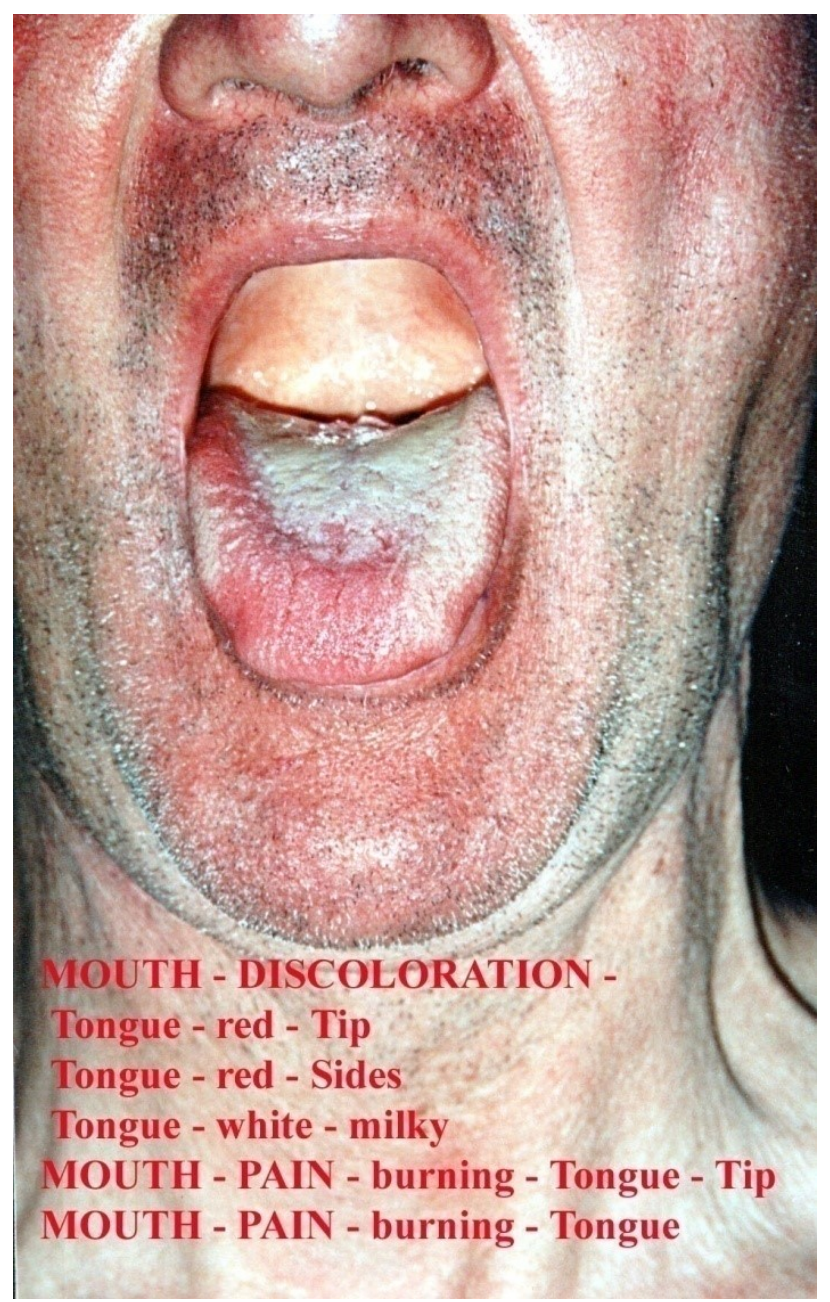

Figure 14
Figure $16(a, b)$ exhibit another configuration: the tongue is slightly deviated to the left, the base is covered by a thick coating; correlation with arcus senilis, in a configuration of Plumbum
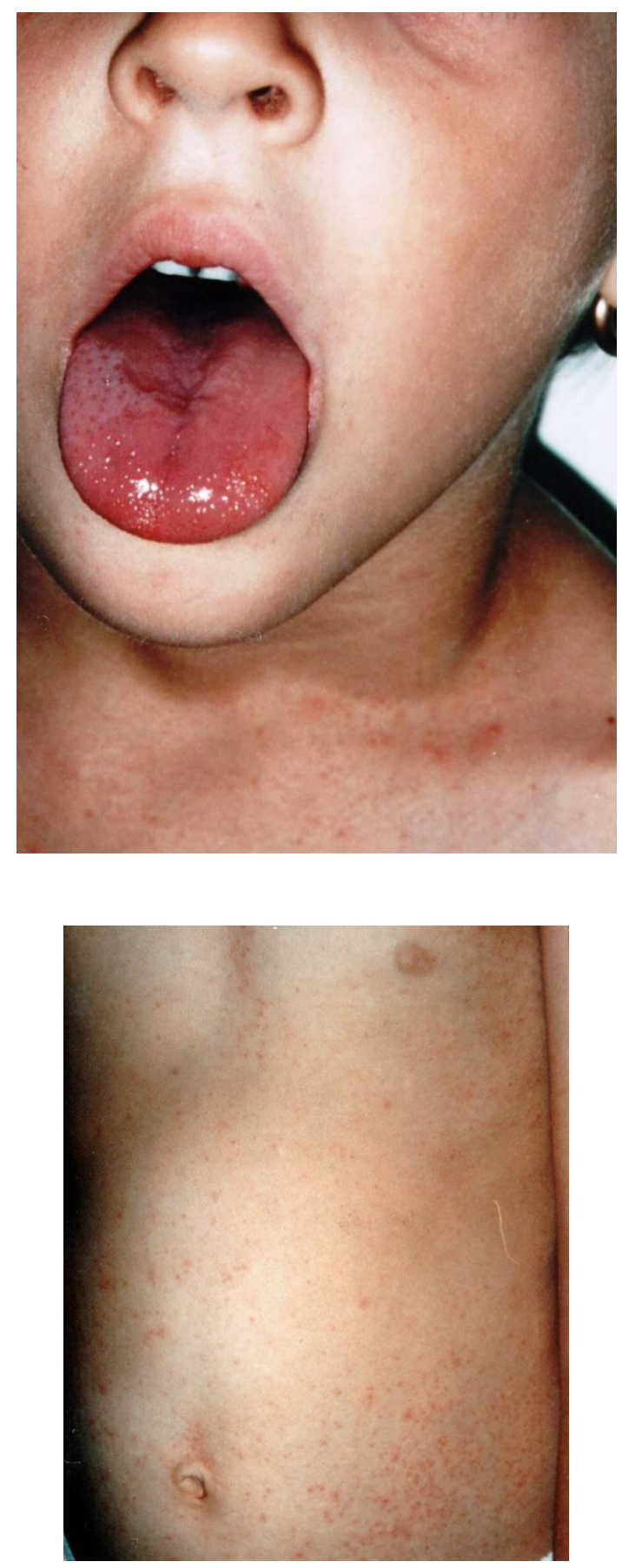

Figure 15(a, b) 

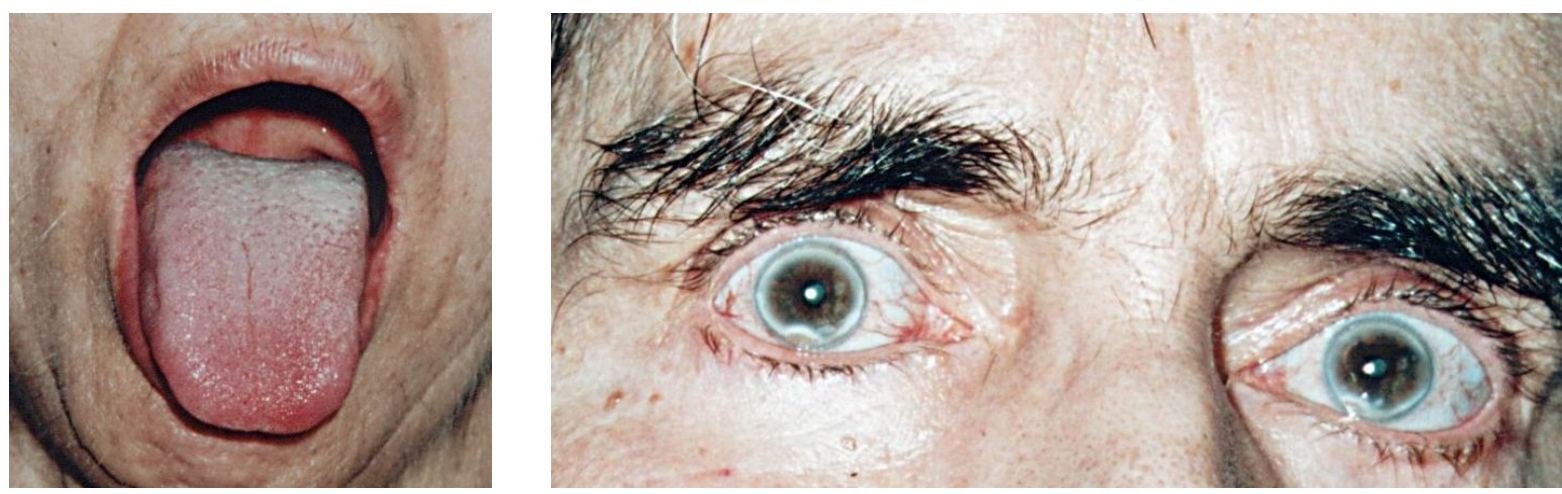

Figure $16(a, b)$

To summarize, there are basically nine species of correlation involving visual signs in homeopathy (Table 1), illustrated in Figures 17-19.Correlation among several signs appearing in a same special area (proximity), composing a "complex sign" peculiar in itself.

\begin{tabular}{|ll} 
Species & \multicolumn{1}{c}{ Correlation } \\
\hline 1 & $\begin{array}{l}\text { Correlation among several signs appearing in a same special area (proximity), composing a } \\
\text { "complex sign" peculiar in itself. }\end{array}$ \\
3 & $\begin{array}{l}\text { Correlation among visual signs and subjective symptoms referred to the same area. } \\
4\end{array}$ \\
5 & $\begin{array}{l}\text { Correlation among different visual signs appearing in different areas (visual Gestalt). } \\
\text { audition, etc.) or instrumentally mediated (laboratory findings, pathological samples, imagery, } \\
\text { etc.) }\end{array}$ \\
$\begin{array}{l}\text { Correlation among symptoms as expressed in anamnesis and the overall configuration of signs } \\
\text { (principle of coherence) }\end{array}$ \\
$\begin{array}{l}\text { Correlation between visual signs and their signification in homeopathic codes (repertory, } \\
\text { materia medica). }\end{array}$ \\
8
\end{tabular}

Table 1. Patterns of correlation of visual signs

The patient presents polymorphous erythema and recurrent stomatitis; the tongue is covered by a heavy yellow-brownish covering and exhibits deep ulcerations on a dysplasia base, cyanosis and depapillation of the tongue and hypersalivation. (Figure 17) 


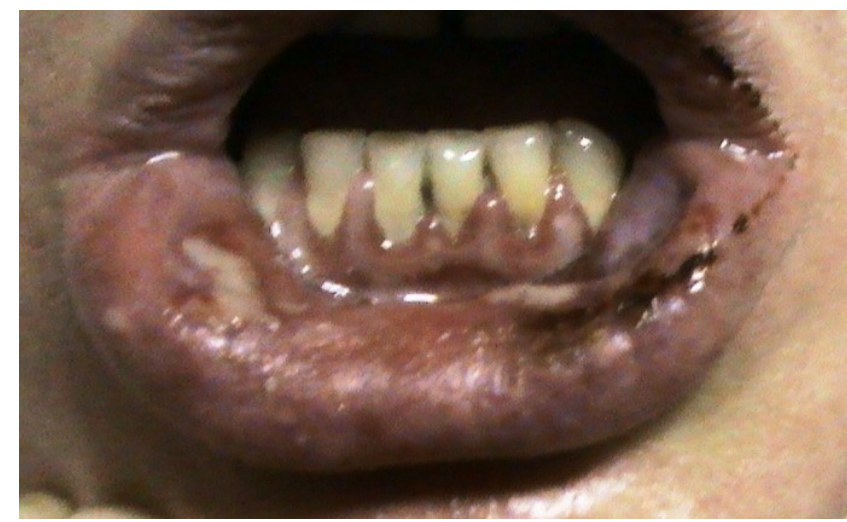

Figure 17

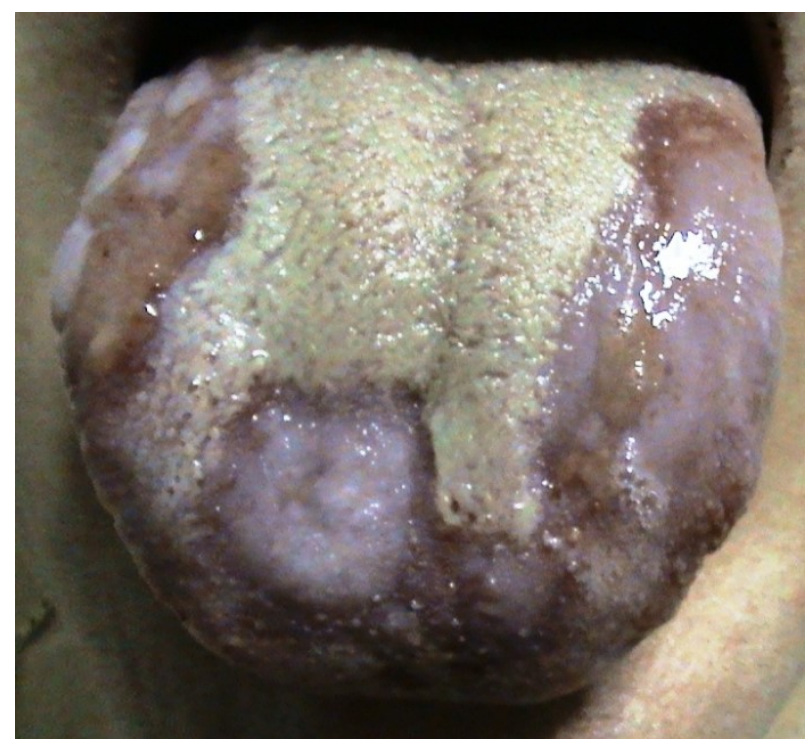

Figure 18

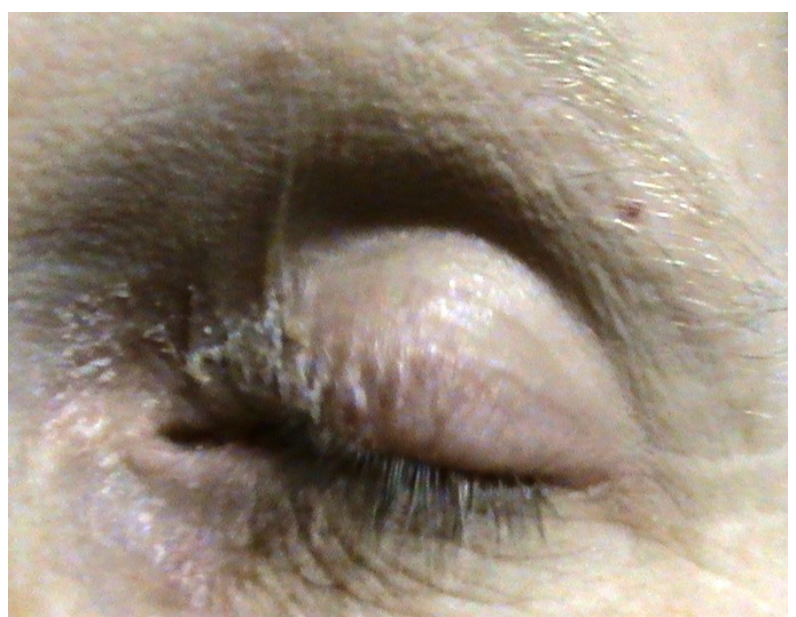

Figure 19

The gums are ulcerated, cyanotic and bleeding, all in correlation with excruciating burning pain. (Figure 18)

The margins of the eyelids exhibit lichenification and desquamation secondary to intense itch. (Figure 19)

This configuration corresponded to Sulphuricum acidum. Correlation with results is shown in Figure $20(\mathrm{a}, \mathrm{b}, \mathrm{c})$ confirming both the choice of the remedy and the hypothesized initial correlation between this configuration and the selected remedy.
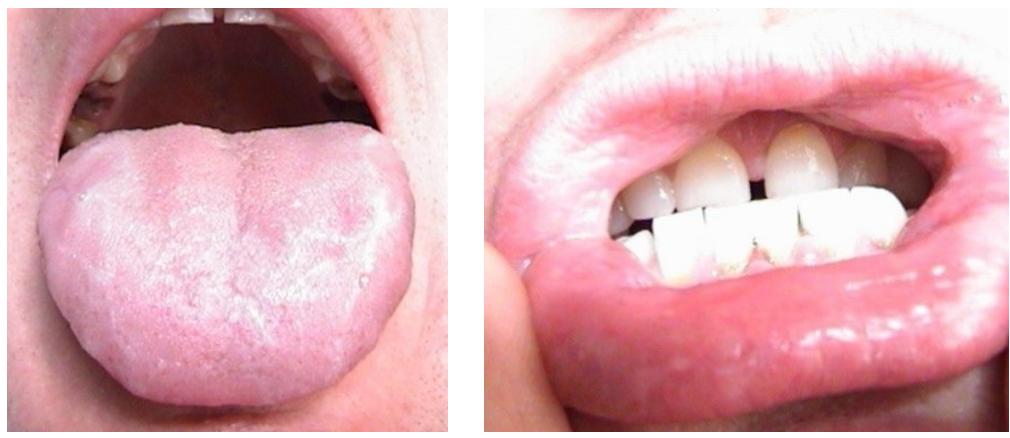

Figure 20

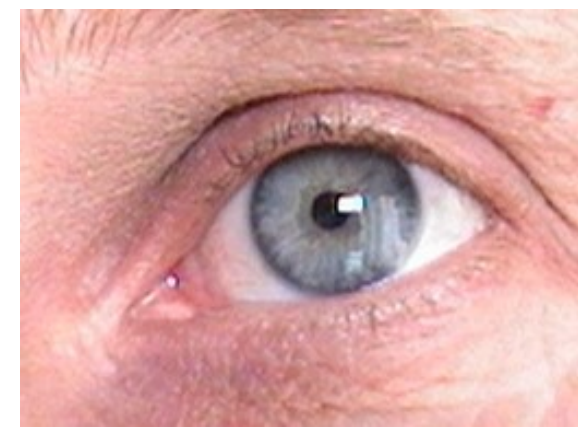

Finally, regarding reintegration, the question to be asked is: how does meaning appear? Let us place as departure point the following configuration of signs (Figure 21). 


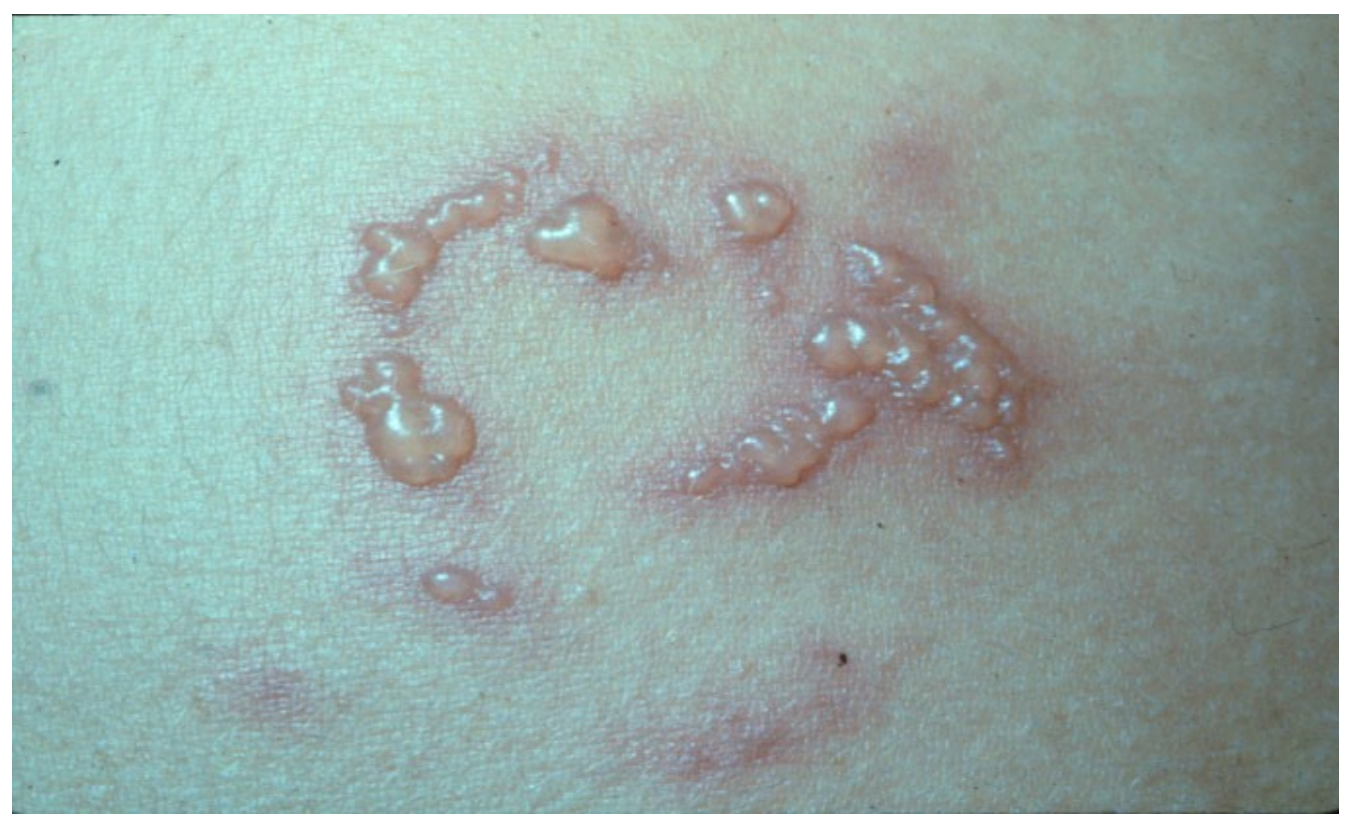

Figure 21

The first feature that must be kept in mind is that signification and meaning are not equivalent terms. Therefore, after a sign is signified as, e.g. in this case /confluent vesicles/ it still lacks a meaning. Meaning is attributed by the receptor of the sign through the insight of the possible totality that signifies the sign in its context. In this regard, a sign is only meaningful when it opens a semiotic field, i.e. a field of possible significations. On the other hand, when the final goal is decision-making and action, as in medicine, in a later stage the semantic field must be constricted.

For a sign in the medical context to acquire meaning it must be defined, qualified and placed within a context, i.e. denotation and connotation must be extended. On the other hand, for the purpose of decision-making, the range of possibilities must be restricted to: 1) those possibly most significant; 2) those most probably therapeutically effective.

Connotation alludes to fields correlated with a sign. Connotative fields do not define signs but open pathways to establish their possible meanings, i.e. "what a sign might mean". In other words, the connotations of sign are a kind of possible explanations or modes of understanding. In the case, possible connotations are "this is due to a toxic substance which forms vesicles" or a disease such as pemphigus.

Denotation, on the other hand, approaches a given sign from its concrete qualifications as its definition, i.e. "this something is such". In our example, "confluent vesicles filled with a clear fluid on an erythematous background itching chiefly in the night".
The boundaries of denotation fields constitute signification; however, this is not yet enough to establish meaning. That is to say, denotation is merely a description of an isolated phenomenon, an extension of its definition according to its actual concreteness. In order for meaning to emerge, it is needed a configuration, i.e. an extension of connotation or that which is intelligible in the sign.

Possible modes of signification include: 1) definition; 2) qualification along modulating axes; 3) inclusion of other signs (configurations); 4) extension of the connotative fields.

The first level of signification relates to the definition of the sign, i.e. the translation of a visual sign into a verbal (linguistic) sign, in our example: /vesicles/ signifies the shift from the normal aspect of the skin to a sign in the medical context.

The second level involves the qualification of the general (normative) sign /vesicles/ into "these" particular vesicles: confluent; on an erythematous background; filled with a clear fluid; appearing in cold weather and fever; itching by heat and in the night.

The next level involves including other signs as to obtaining a configuration. In our example, the patient also exhibited a white covering on the tongue, extending all over its surface except the tip and the middle line (Figure 22). 


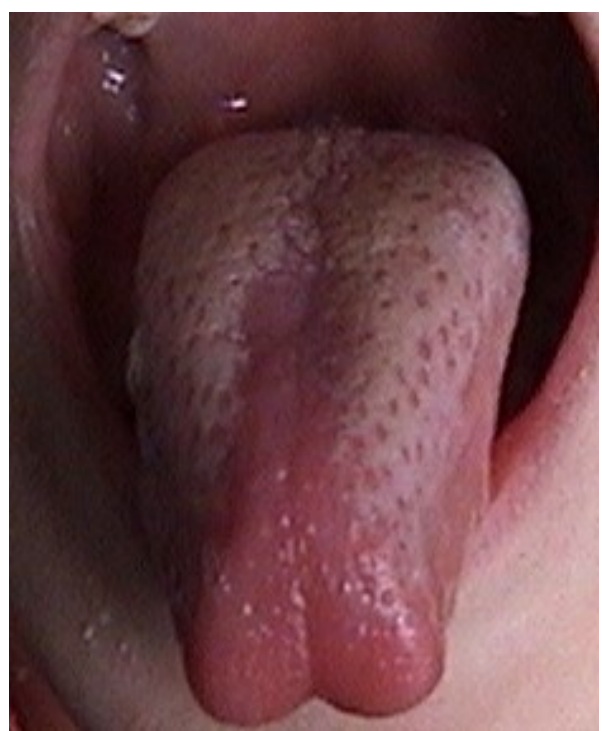

Figure 22

The fourth and final level of signification involves the extension of the connotative field, which can be performed, e.g. with the assistance of other disciplines. In this way, toxicology teaches us that this kind of vesicles appears after external contact with poison ivy (Figure 23); microscopic anatomy shows that vesicles are due to detachment of epidermis (Figure 24).

In this way, the final meaning of this sign is "Rhus toxicodendron".

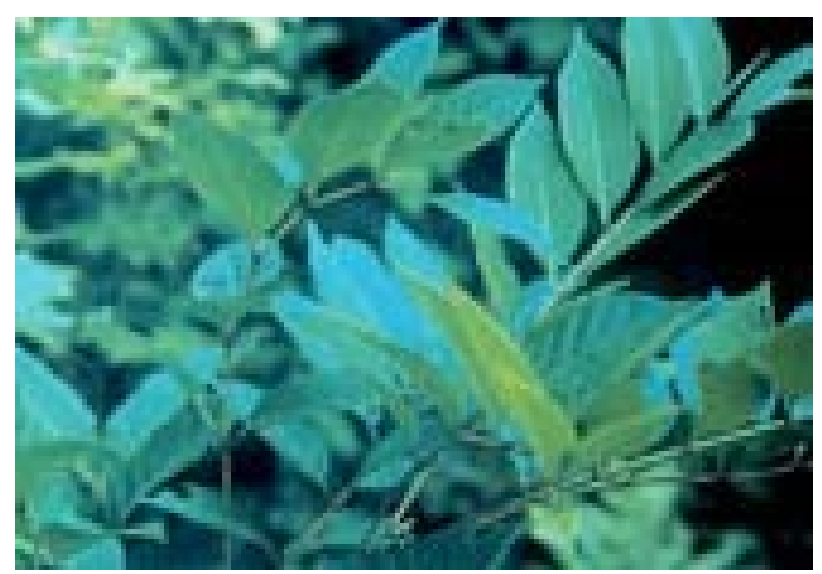

Figure 23

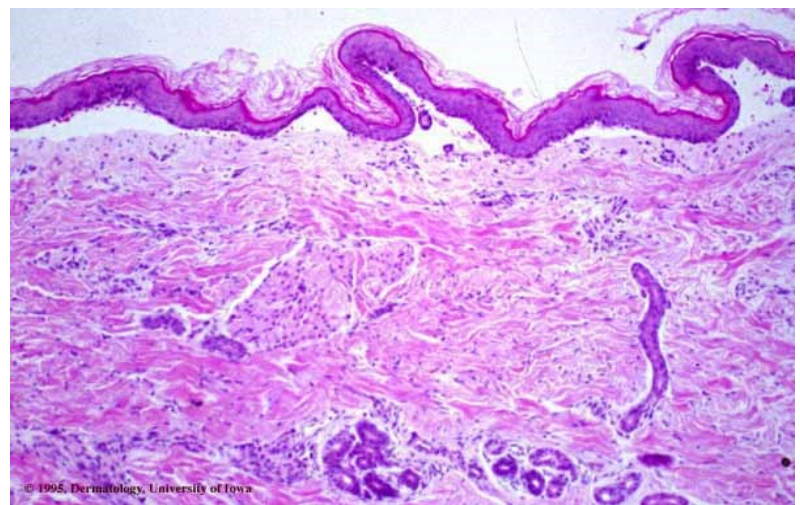

Figure 24

Let us approach now integration from the opposite side and look closer at the notion of configurations. It is universal homeopathic knowledge that for a same disease, different patients exhibit different signs and symptoms and consequently resulting in different remedies.

However, how does one and the same remedy express itself in different diseases?

In fact, we cannot "identify" remedies: clinical reality presents us only indicators, i.e. signs pointing to some remedies. Rubrics in repertories help in restricting the field of possible remedies, however, what is truly effective in restricting consistently this field are configurations of signs rather isolated ones. Configurations can be simply defined as "groups of signs that appear together", that are intrinsically connected.

To identify configurations, a useful tool is to know the picture of intoxications, e.g. when one knows how atropine intoxication looks likes, it is easier to recognize a configuration of Belladona. Intoxications are particularly relevant, as in this case signs and symptoms never appear isolated, but systematically in complexes (configurations) derived from the physiopathologic processes triggered by the toxic. Let us present one such configuration (Figure 25). 


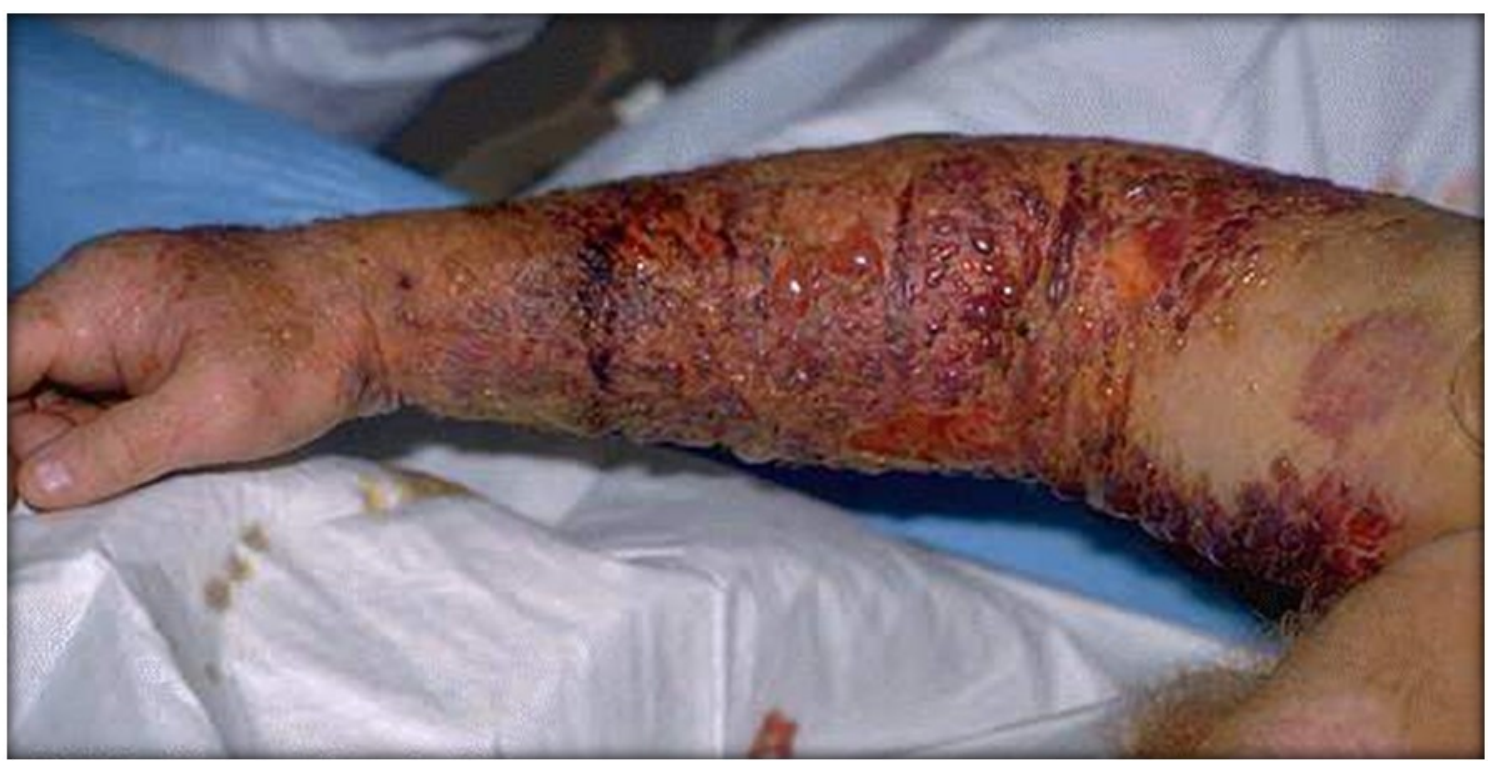

Figure 25

The elements associated in this configuration are: 1) large boils; 2) swelling; 3) ecchymosis; 4) cyanosis; and 5) lymphatic affection. The cause of this picture is a toxic substance which produces: 1) microthrombosis with 2) laceration of the superficial layer of the skin, which thus is detached forming boils; 3) disturb of coagulation; 4) disturb in the microcirculation, predominantly venous (cyanosis); and 5) edema. It can be a disease, as e.g. bullous pemphigo, it, it can be an intoxication. It is a snake bite. From the homeopathic clinical viewpoint this is extremely relevant: a similar configuration in a patient points to a snake-derived remedy, e.g. Lachesis, as in the following cases.

Case 1. Patient consults with severe congestive heart failure; is in very poor general state, with dyspnea and very irritable. He had spent the last three weeks hospitalized, but there was no improvement; on auscultation he presented signs of chronic pulmonary edema and he also had ulcers in the legs (Figure 26).

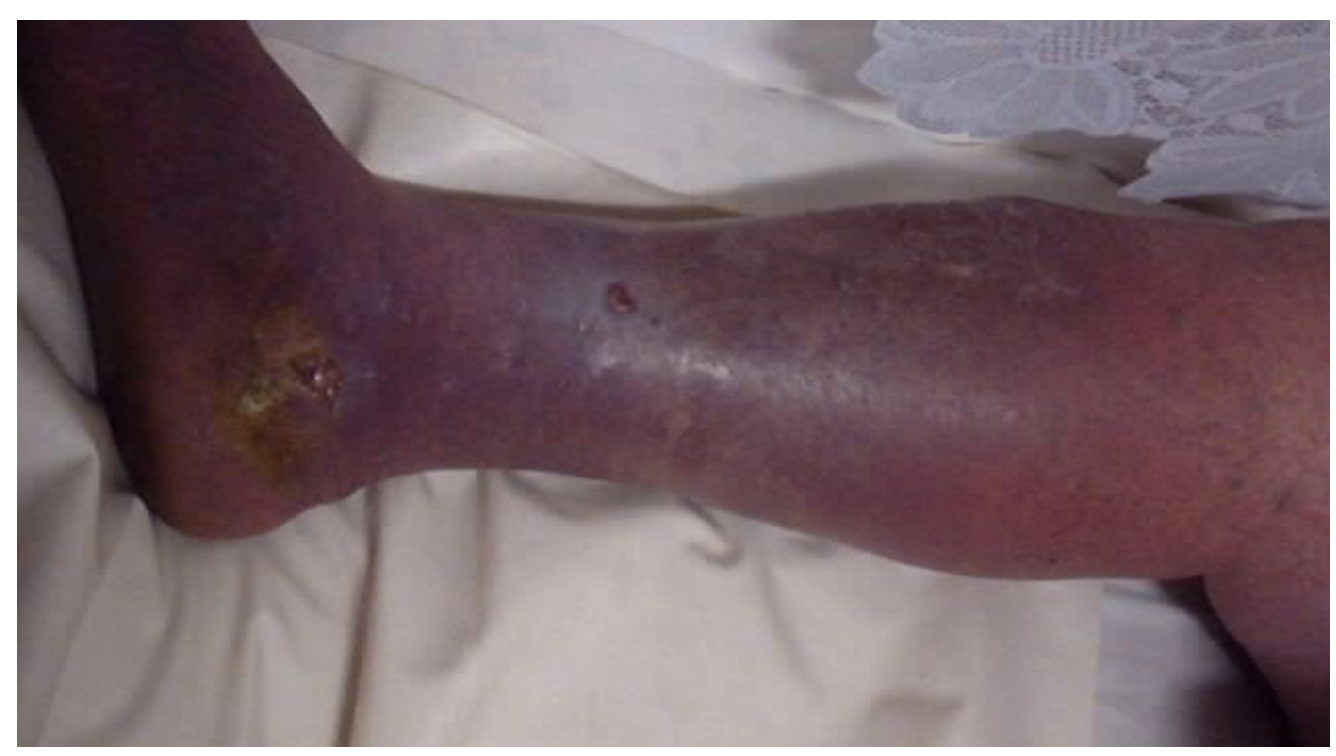

Fgure 26

Clinical picture was composed of characteristic signs of the disease (cyanosis, peripheral edema) as well as of signs of this individual-in-disease: deep blue discoloration of the distal extreme of the lower limbs with congestion in the proximal one and ulcers. His face presented similar signs (Figure 27): overall congestion and infiltration, deep-blue cyanosis (lips, nose, ears) and venectasis on the cheeks. 
However, a closer look on the lips (“zoom-in” model) showed, in fact, an alternation between congestive and cyanotic areas (Figure 28), the same pattern as in the lower limbs.

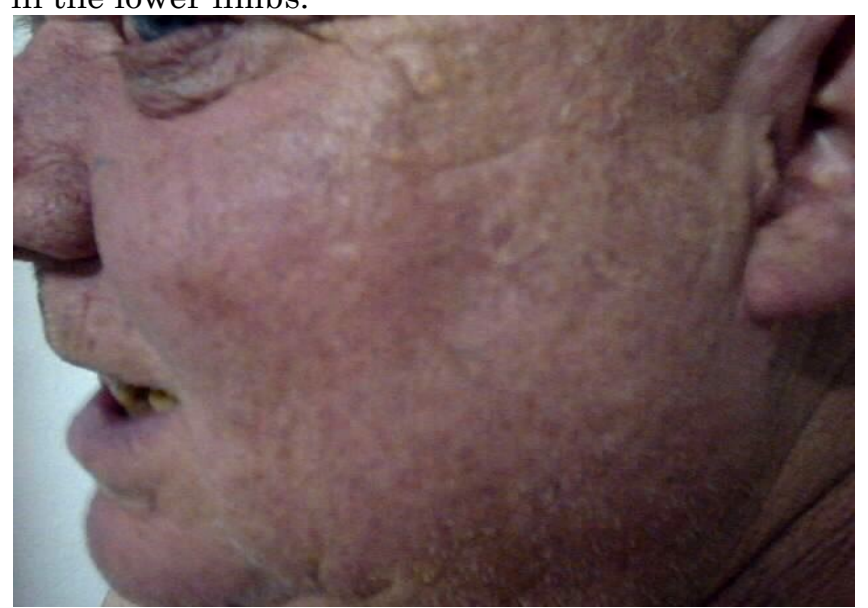

\section{Figure 27}

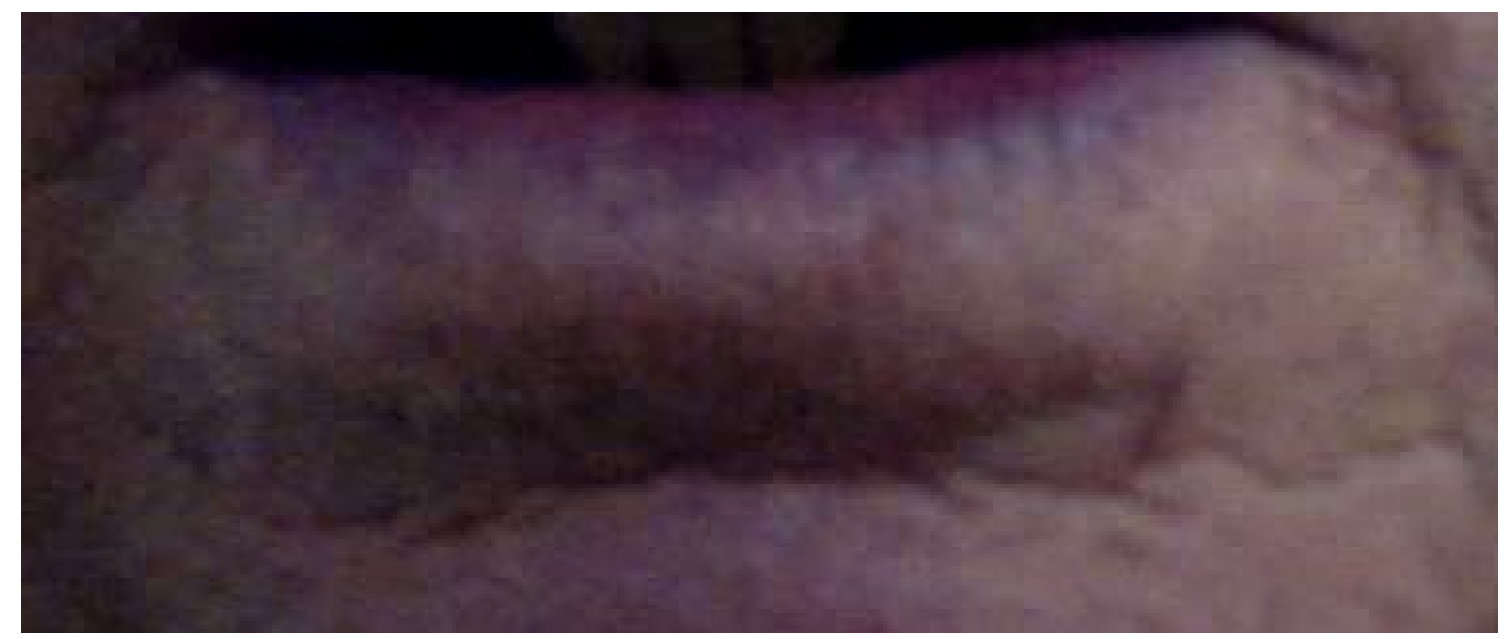

Figure 28

Venectasis also on the back (Figure 29), which is an extremely rare localization for this phenomenon, therefore, a peculiar sign.

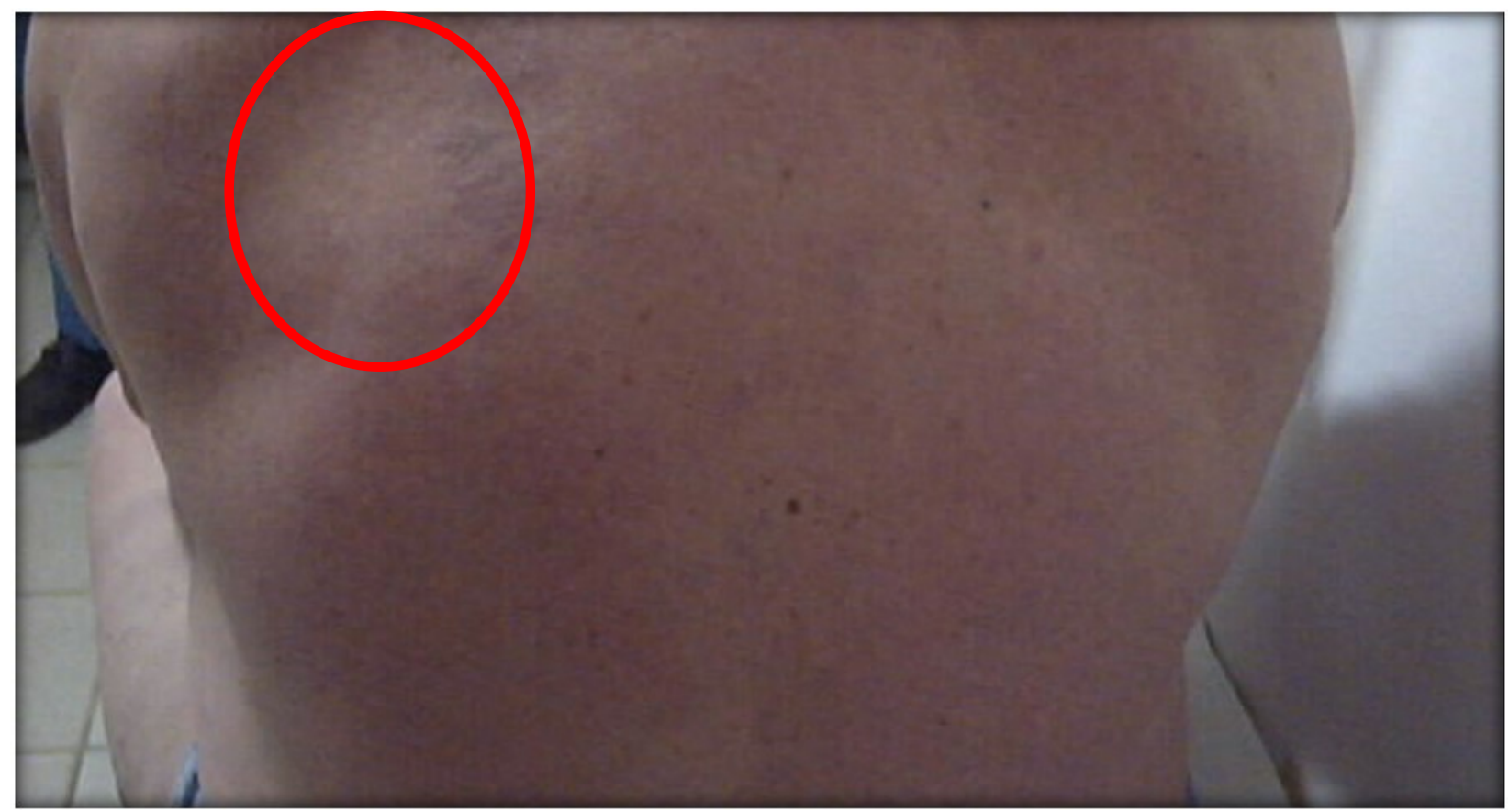




\section{Figure 29.}

Coagulation disturb led to bleeding in the sites of venopuncture (figure 30)

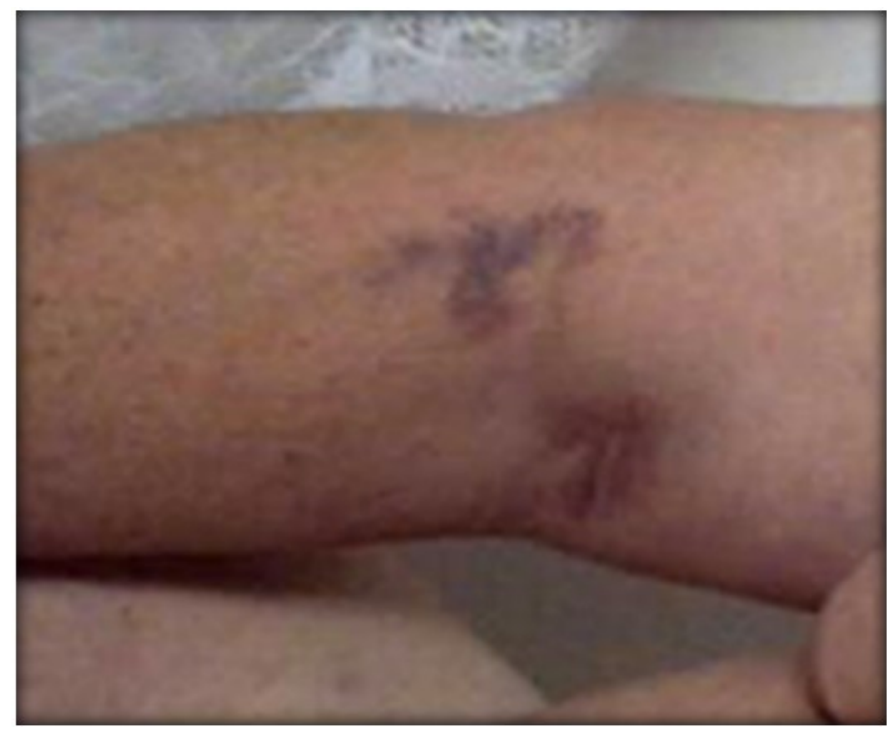

Figure 30

The tongue was bifid, deviated to the left side, exhibited dramatic cyanosis and a toxic white coating (Figure 31).

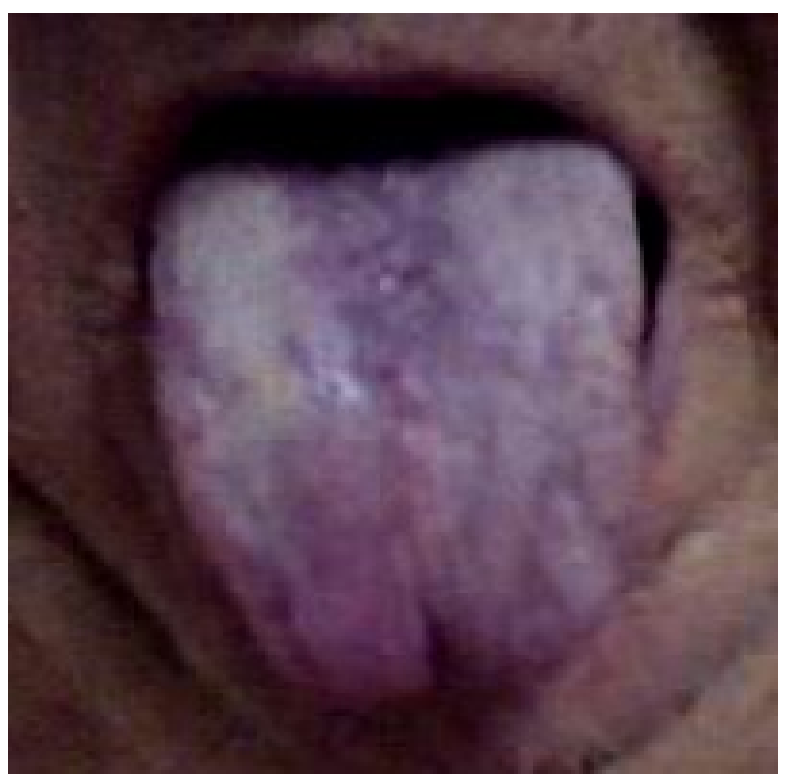

Figure 31

A closer look ("zoom-in") on the ulcers showed once again an alternation of red congestive and blue cyanotic small areas as well as a humid secretion (Figure 32).

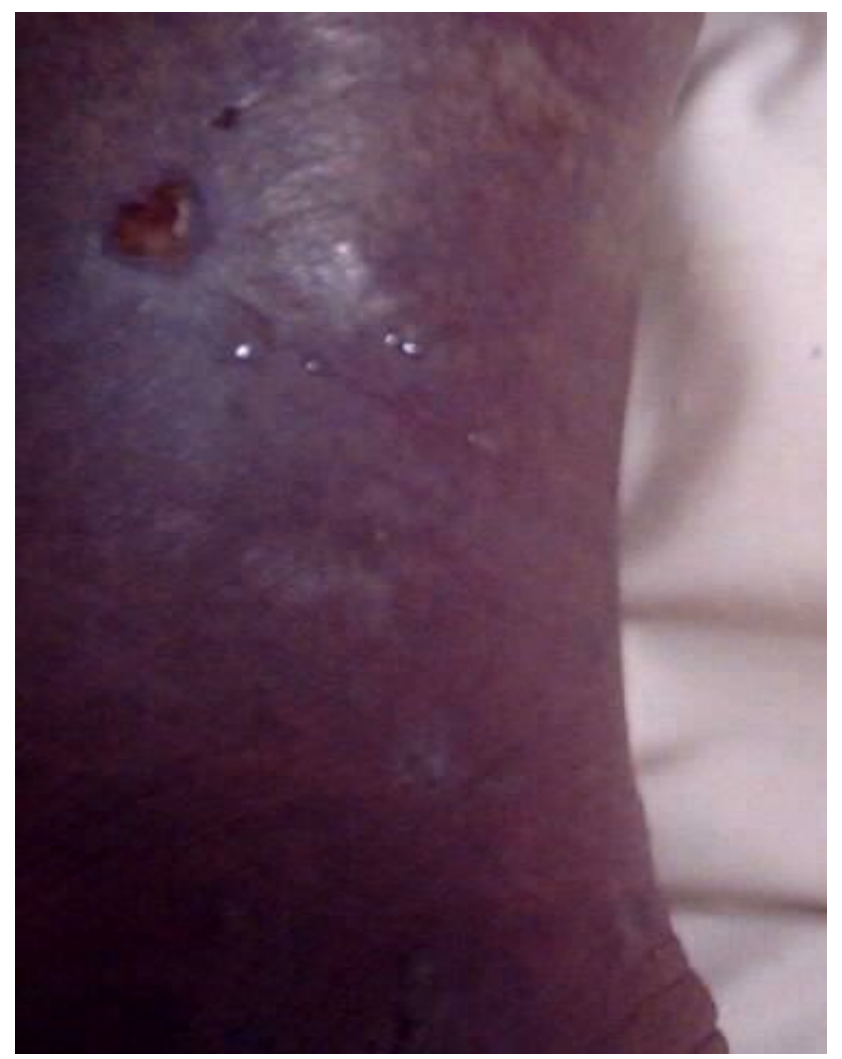

Figure 32

Ulcers were atrophic: lack of granulating tissue. (Figure 33) It is useful to remind here that not too many homeopathic remedies have been related to atrophic ulcers (Carb-an, Carb-v, Kali-bi, Lach, Merc and some acids, such as Ph-ac and Sul-ac).

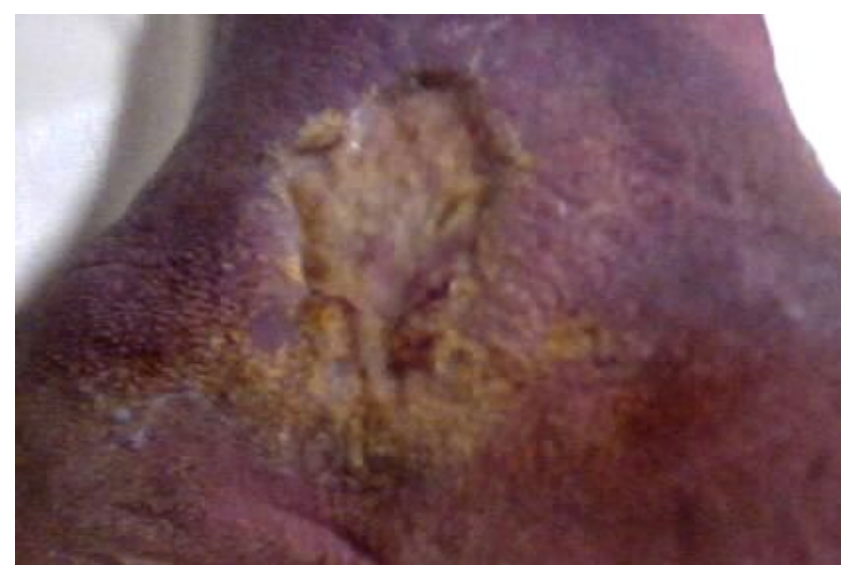

Figure 33 
A coherent configuration leads to individualization: in each localization and lesion the same phenomena are present: alternation of congestion and cyanosis in small areas; toxic appearance; infiltration and swelling; ecchymosis (disturb of coagulation); microthrombosis and venous stasis. Prescription of
Lachesis in low dilution $(9 \mathrm{cH})$ led to improvement of circulation, visible and also through auscultation, with reduction of pulmonary edema; improvement of dyspnea and weakness as well of the mental symptoms. (Figure 34)
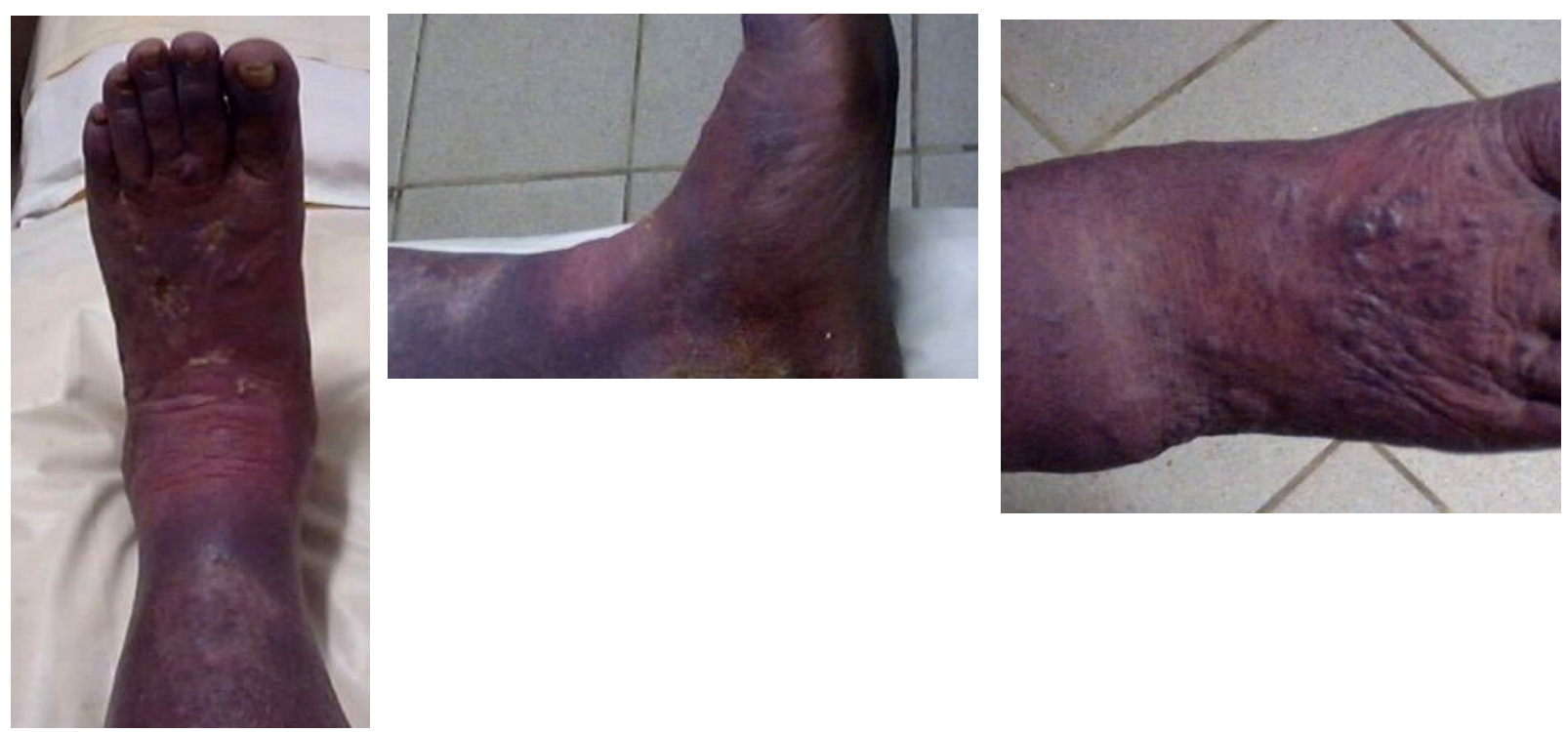

Figure 34

These signs, which appeared dramatically in the patient above, can also be reencountered in the case of this 9 year-old girl suffering from psoriasis. At first sight, lesions look typical of the disease (Figure 35)

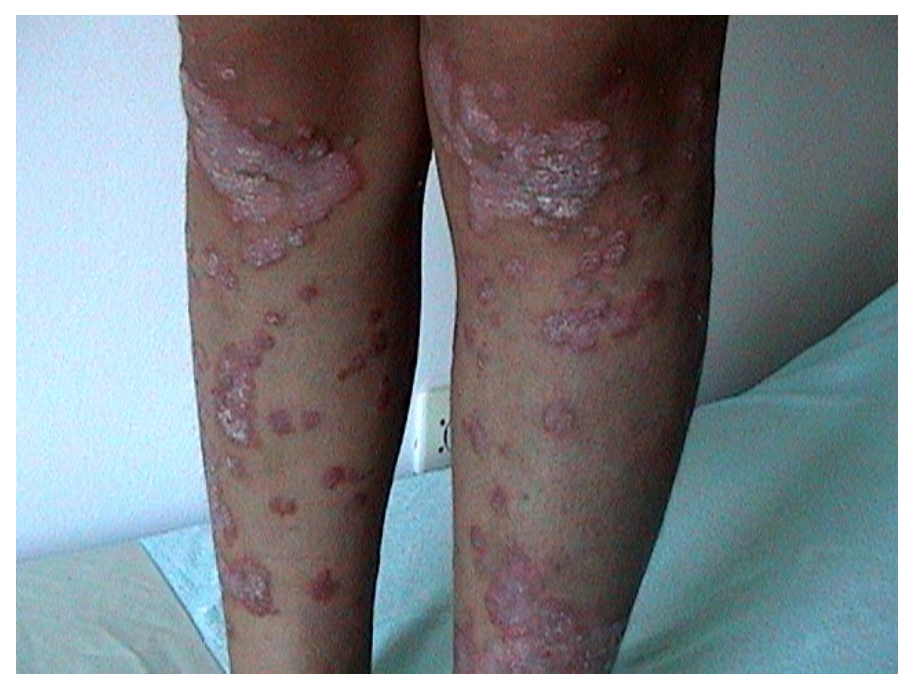

Figure 35
However, a closer look ("zoom-in") reveals an alternation of congestive and cyanotic in small areas, completely unrelated to the physiopathology, but peculiar to this particular patient. (Figure 36) There are also signs of edema (Figure 37)

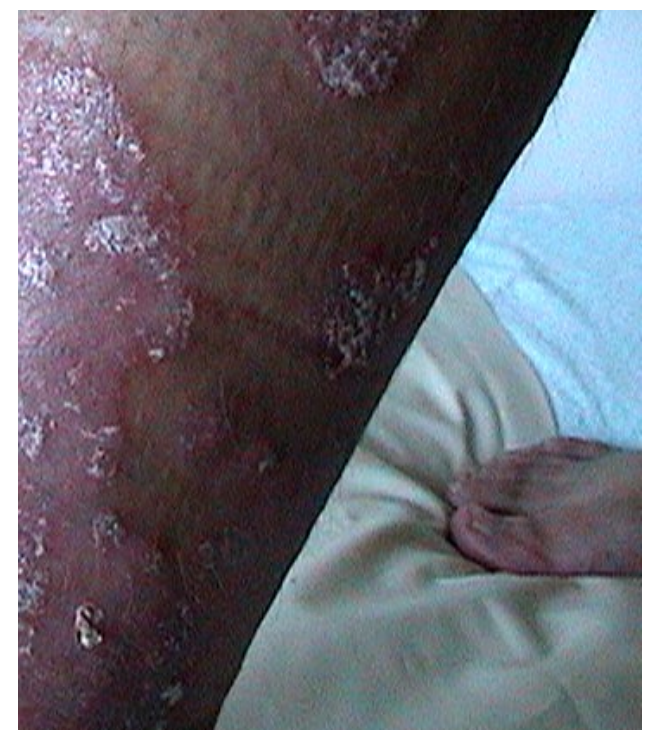

Figure 36 
And finally, once again the tongue is bifid, blue and deviated to the left side; the lips present the alternation of blue and red discoloration and the face is congested. (Figure 38)

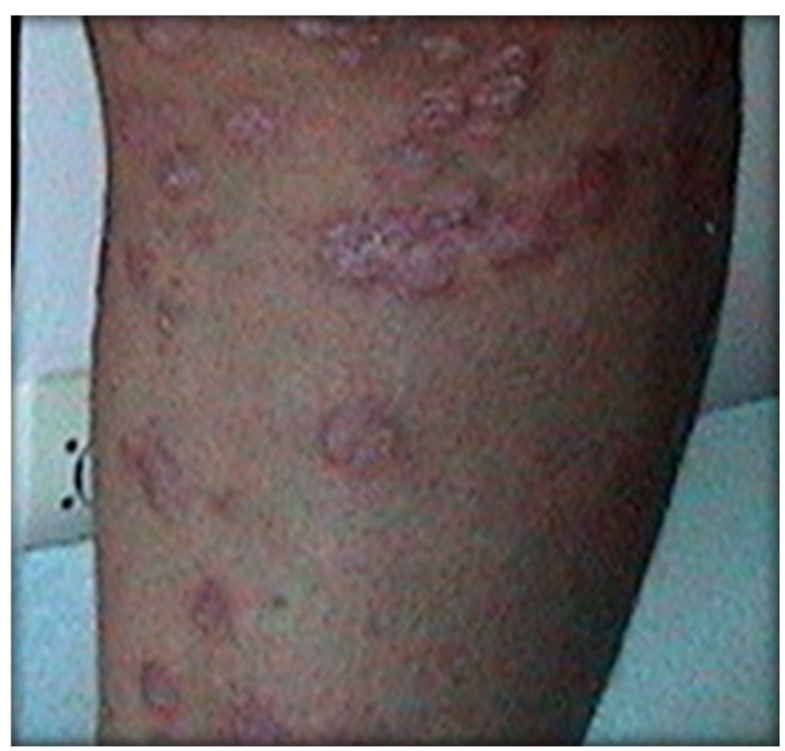

Figure 37

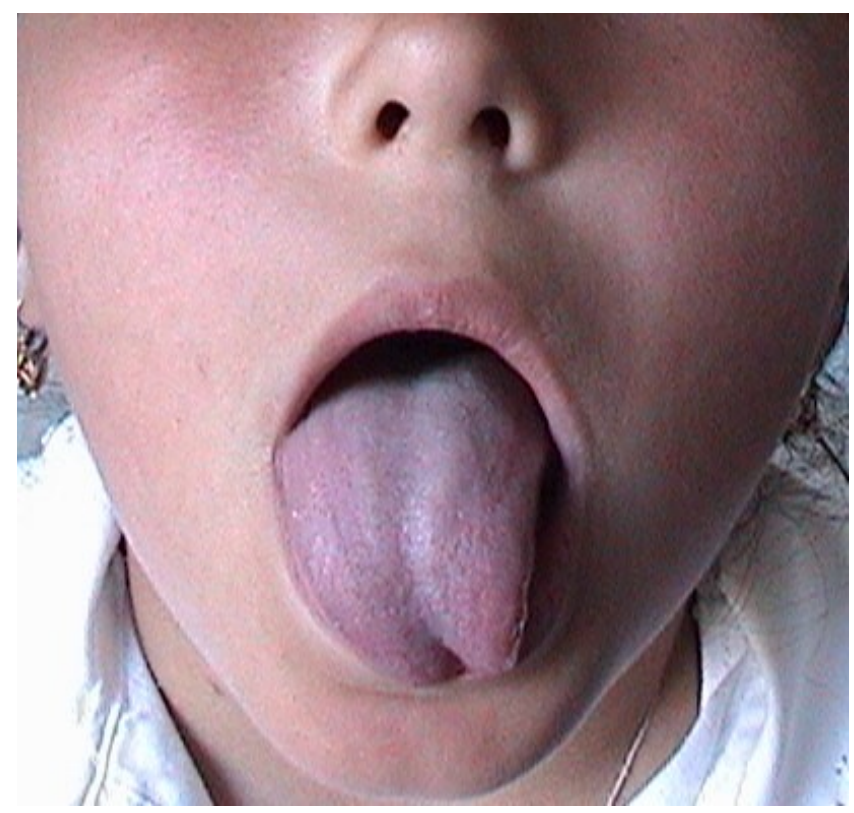

Figure 38

\section{Discussion}

Integration of signs requires addressing the sources of those that can be recognized in homeopathic clinical practice. Shortly, sources are the homeopathic materia medica, toxicology and clinical experience.

Regarding the homeopathic (pure) materia medica, or record of proving-based signs, signs can be noticed by provers themselves or by the proving director. In both instances it is a matter of an objective (auto or hetero) observer which notices phenomena and reports them summarily. However, from the theoretical standpoint there is not one single criterion enabling one to perceiving anything but a sign by itself. At this level there is no way to know whether a sign is the direct effect of a substance on an individual, the impact of a substance on a sensitive individual triggering particular effects specific to this particular individual or if it a matter of an unspecific stimulus eliciting an individual response associated to the particular pattern of idiosyncrasy of the prover. In other words, causally, we are dealing with three different possibilities: the effect of a substance, the peculiar sensitiveness of an individual to a substance and decompensation of the individual's state due to an unspecific stimulus; however, the phenomenal expression is identical: this one specific sign.

For this reason, in homeopathic medical semiotics, indifferently from the precedent cause (i.e., that which makes a sign appearing in a given proving), signs must be taken as such. The relevant feature here is not why a sign appears, but the fact it appears. Therefore, what a proving teaches is that a given sign has appeared in a prover, but it cannot establish whether the proved substance was its direct (material) cause, its efficient (triggering) cause or a mere conjunctural event.

The same happens with actual patients. Regarding any sign exhibited by a patient, we can only know that such sign does exist, but simple observation does not allow one to infer that it was caused by the ongoing disease, that it is an idiosyncratic reaction to the disease or that it expresses the patient "such as he or she is".

Shortly, both proving and diseases result in signs and associations of signs. When signs appear together in a given individual in a definite moment, they constitute a configuration of signs. Such configurations are descriptors of a semiotic field which comprises the totality of signs determined by the informational input conveyed during a proving (determined, conditioned, real). Disease, as a nosologic entity (abstracted and abstract) is a semiotic field defined by a given configuration of signs. However, from this perspective, the individual is methodologically excluded from the notion of the disease: the configuration of the signs of the disease is abstracted from the actual totality of existing signs according to predefined signifiers, whereas the signs depending from the particular concrete individual are excluded, as non significant.

As it is known, one of the premises of homeopathy is precisely the consideration of particular individual signs. This kind of signs is particularly valued as they possess a potential of signification leading to therapeutic decision-making due to the existence of semiotic fields where each particular sign can be 
included. In this way, the classical homeopathic notion of the "totality of symptoms" can be seen as its very integrative notion, this is, as a semiotic field allowing for the recognizance of signifiers well beyond the level of disease. Configurations of signs signify semiotic fields.

A sign can become particular through: 1) determination: structural (depending on the composing elements) or qualitative (the way how it is manifested); and 2) its situation within a context, e.g. "frontal headache accompanied by diarrhea after eating sweets". In this way, we do not deal anymore with "simple signs" but with determined signs, individualized according to their own qualities (localization, appearance, modalities, etc.) or to their relationship with an individual context, whether pathological or not.

The signs "of disease", corresponding to a preestablished semiotic field, become particular through the identification of their concrete qualities; this process widens the initial semiotic field, leading to the identification of the "individual disease". In this context, the signs presented by an individual and that are not related of disease can belong to one of the following classes: 1) signs that can be included in semiotic fields of "disease", as e.g. concomitant disease; 2) signs that cannot be included in the semiotic fields of disease. The latter are signs depending on the individual as such, i.e. that express the individual and for this reason, the signified is the individual.

Inclusion of a sign into the semiotic field of a disease is a mental construction: reality only presents us a set of concrete signs; semiotic fields of disease are signifiers appearing in pre-established configurations. Regarding signs that cannot be included in semiotic fields of disease, whereas conventional medicine dismisses them completely, homeopathy takes them into account, as the notion of "totality of symptoms" allows for dynamic semiotic fields - rather than fixed, as in conventional medicine - which may include a variable number of signs.

Fixed semiotic fields oriented towards disease are well delimitated and stable and correspond to a definite configuration of signs that, thus, become socalled diagnostic criteria. Relationship between two or more fixed semiotic fields is divergent: each includes exclusively the signs typical of a given disease and excludes all others; such fields "repel" one another and fragment the continuity of the whole.

Dynamic semiotic fields, on the other hand, allow for an integrative model, where all expressing in any possible way an individual is a part of. Such fields are poorly defined, even fuzzy, and on the other hand they are convergent, as their final signified is a totality: signs "attract" one another and act reciprocally to maintain a configuration. In an integrative model, both the signs of disease and the signs unrelated to disease may become significant, as well as both verbal and non verbal signs. Each perceptible sign can lead to disease (entity), the individual-in-disease or the individual such as he is. Accordingly, as a function of their signifier, visual signs can indicate disease (entity), the individual-in-disease or the individual such as he is.

Visual signs of the individual-in-disease are unspecific, however only inasmuch as they are not determinate in their particularities, which is done through the identification of their morphological characteristics ("zoom-in model"), their correlation with the wider context ("zoom-out model") and their correspondence with verbal signs. Although they chiefly are signs of individual disease, their semiotic field is widened through their association with: 1) general conjunctural signs (when, why, etc.); modulating signs (modalities of emergence, aggravation, amelioration, etc); and 3) concomitant signs (dependent of convergent semiotic fields). In this way, what is apparently unspecific (e.g. signs of psoriasis) may become highly specific through a more accurate description and placement within the actual context of the patient. In fact, "not all psoriasis cases are alike", as neither "all erythematous pharyngitis" are.

Visual signs indicating the individual as such express his or her way of being, i.e. who he or she is, in disease or outside disease. Their determinants are blurry, for this reason they must be taken such as they appear. Signs arising from the genetic makeup, the personal history, life-style, conjunctures and conjectures, all of them are integrated in a stable individual configuration representing the morphological and functional pattern of idiosyncrasy of each singular patient.

To summarize, we operate among two stable configurations and two unstable configurations of signs. The first two are the configuration of signs in disease, as a product of the epistemological process of construction of species of disease, and the configuration of signs expressing an individual; the other two are the configurations of signs of an individual-in-disease and the configurations of signs of the disease(s) of an individual. From this crossroads that emerges the need of visual semiology in homeopathy: because it addresses both stable semiotic spheres, the configurations of disease as it appears and the configurations of the individual. Moreover, it allows for an insightful incursion also into the unstable spheres of the individual-in-disease and the disease of the individual.

Vision also "talks" and it tells us what a patient expresses through visual signs through a different 
language, a transverbal language in which the final signified is the individual as such, whether in disease or not. A language where signs are sent, but they still await a receptor.

Patients "talk" through gestures, which become signs only when there is a receptor. Through gestures, they "talk" about them, they "tell" how they are in the present moment, they let us know what they are feeling, they ask questions, they answer ours. Also their demeanor expresses their present state or their way of being, their psychological characteristics, reactions to internal and external stimuli, and the relationships to others. And as it was shown in this paper, even the slightest physical sign may reveal unsuspected aspects of the individuality of patients.

\section{Conclusions}

Application of the model proposed results in highly qualified and individual visual signs that bring new elements to reconsider the role of objective signs in homeopathic practice. The foundations of a method of seeing in homeopathy include the following items:

1) Principle of difference: grosso modo, signs relevant in homeopathy are those that represent "something different" - exaggerated, characteristic, rare, special, strange - in two levels of reference: disease and the individual. Shortly, something differs from the typical picture of the disease or the way of being of the individual.

2) Recognition of signs implies initially the analysis of the context within which something different appears, and then "zoom-in" and approach signs in full detail: the signs emerges as a break in the continuity of what is considered "normal", usual, typical or expected; variation refers, thus, to the context and the norm.
3) Discontinuity applies to something differing from the rest having a same structure or belonging with a same context. Variation regarding a norm happens when something seen differs from the "normal" image we have in the mind, according to medical codes of normality and pathology. Variation regarding to context warns us that something which is normal in a given context may not be in another.

\section{References}

[1] Jurj G. Decision making and semiotics: A view in homeopathy. Int J High Dilution Res [online]. 2008 [cited 2009 June 01]; 7(23): 103-112. Available from: http://www.feg.unesp.br/ ojs/index.php/ijhdr/article/ view/270/346.

[2] Santaella, Nöth W. Imagem: cognição, semiótica, mídia. São Paulo: Iluminuras; 1998.

[3] Jurj G. Understanding homeopathy by images. [CDRom]. Timisoara: Jurg G.; 2001.

[4] Jakobson R. Linguistics and poetry. In: Sebeok TA, editor. Style and language. Cambridge, Mass: MIT Press; 1960. 350-377.

[5] Peirce CS. Collected Papers, editors. Hartshorne C \& Weiss P (vols. 1-6); Cambridge, Mass: Harvard University Press; 1931-1958.

[6] Peirce CS. Collected Papers, editors. Burks AW (vols 7-8.) Cambridge, Mass: Harvard University Press; 1931-1958.

[7] Peirce CS. Writings of Charles S. Peirce: a chronological edition. Houser N, editor. Bloomington/Indianapolis: Indiana University Press; 1981.

\section{(c)) EY-NC-ND Licensed to GIRI}

Support: authors declare that this study received no funding

Conflict of interest: authors declare there is no conflict of interest

Received: 15 Apr 2009; Revised 10 Jun 2009; Published: 30 Jun 2009

Correspondence author: Gheorghe Jurj, relujurj@gmail.com

How to cite this article: Jurj G. A Method of Seeing in Homeopathy: Methodological Foundations of Project "Understanding Homeopathy by Images". Int J High Dilution Res [online]. 2009 [cited YYYY Month dd]; 8 (27): 53-69. Available from: http://www.feg.unesp.br/ ojs/index.php/ijhdr/article/view/333/386. 